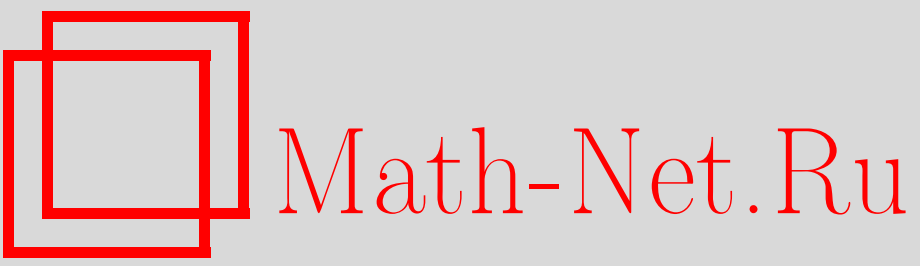

В. А. Васильев, Топология наборов плоскостей и их дополнений, УМH, 2001, том 56, выпуск 2, 167-203

DOI: https://doi.org/10.4213/rm384

Использование Общероссийского математического портала Math-Net.Ru подразумевает, что вы прочитали и согласны с пользовательским соглашением

http://www.mathnet.ru/rus/agreement

Параметры загрузки:

IP : 54.197 .217 .227

26 апреля 2023 г., 15:45:43 


\title{
ТОПОЛОГИЯ НАБОРОВ ПЛОСКОСТЕЙ И ИХ ДОПОЛНЕНИЙ
}

\author{
В. А. ВАСИЛЬЕВ
}

\begin{abstract}
Статья представляет собой краткий толковый словарь понятий и методов, связанных с топологической теорией наборов аффинных плоскостей, включая группы кос, конфигурационные пространства, порядковые комплексы, стратифицированную теорию Морса, симплициальные разрешения, комплексы графов, кольца Орлика-Соломона, комплекс Сальветти, матроиды, двойственность Спеньера-Уайтхеда, подкрученные группы гомологий, теорию монодромии и многомерные гипергеометрические функции.

Делается упор на максимальную геометричность изложения, намечаются приложения и аналогии в дифференциальной топологии.

Приводятся некоторые недавние результаты теории.

Библиография: 96 названий.
\end{abstract}

\section{СОДЕРЖАНИЕ}

$\S 1$. Введение

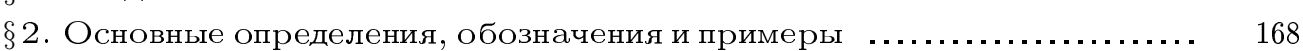

$\S 3$. Основной пример: кольцо когомологий группы крашеных кос $\quad . . . . . .170$

$\S 4$. Кольцо Орлика-Соломона и когомологии дополнения к набору комп-

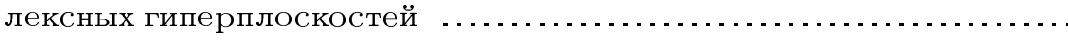

$\S 5$. В какой степени топология дополнения определяется размерностными данными: сводка результатов ............................ 172

$\S 6$. Порядковый комплекс частично упорядоченного множества. Формула Горески-Макферсона ....................................

$\S 7$. Симплициальные разрешения и формула включений-исключений. Спектральная последовательность Майера-Вьеториса и ее мо-

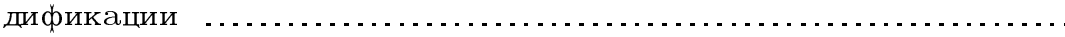

$\S 8$. Гомотопический тип объединения афффинных плоскостей и стабильный гомотопический тип его дополнения

$\S 9$. Примеры: разрешения важных наборов плоскостей. Комплексы связных графов и гиперграфов

$\S 10$. Комбинаторная реализация классов когомологий дополнений к на-

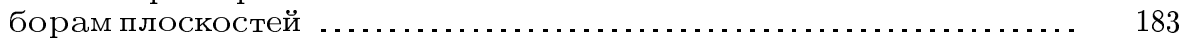

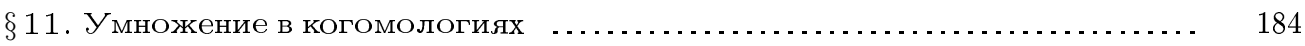

$\S 12$. Комплекс Сальветти для комплексифицированного набора вещест-

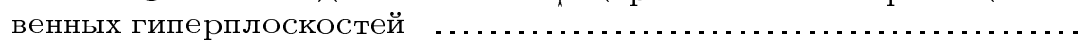

$\S 13$. Гомологии дополнений к наборам плоскостей с подкрученными коэфффициентами. Резонансы 


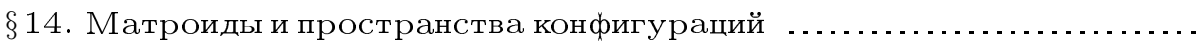

$\S 15$. Приложения в интегральной геометрии: общие гипергеометричес-

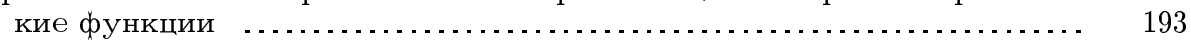

$\S 16$. Что, если набор плоскостей бесконечен $\quad$....................... 196

$\S 17$. Приложения и аналогии в диффференциальной топологии .......... 198

Список литературы ............................................... 200

\section{$\S$ 1. Введение}

Конечные наборы аффинных плоскостей в $\mathbb{R}^{N}$ или в $\mathbb{C}^{N}$ (в англоязычной литературе они называются plane arrangements) образуют замечательньй класс алгебраических множеств. Действительно,

1) в их исследовании переплетаются топология, комбинаторика, линейная алгебра, теория представлений, алгебраическая геометрия, теория сложности вычислений, математическая физика и теория дифференциальных уравнений;

2) они являются прекрасным полигоном для методов и мотивировок, имеюших очень далекие обобщения в этих областях;

3) они дают удачную элементарную визуализацию абстрактных алгебраических и комбинаторных понятий и конструкций.

Формулы, конструкции и теоремы, однажды появившиеся в этой теории, возникают затем вновь и вновь в довольно далеких областях и проблемах.

Один из основных вопросов теории состоит в том, насколько топологические свойства объединения нескольких плоскостей (и дополнения к этому объединению) определяются формальньми данными, т.е. информацией о размерностях всех поднаборов плоскостей.

Мывоспользуемся этой задачей, чтобыпродемонстрировать такие понятия и методы, как группы кос, конфигурационные пространства, порядковые комплексы, стратифицированная теория Морса, симплициальные разрешения, комплексы графов, кольца Орлика-Соломона, матроиды, двойственность Спеньера-Уайтхеда, подкрученные групы гомологий, теория монодромии, гипергеометрические функции и т. д.

Имеется несколько хороших изложений теории наборов плоскостей или различных их аспектов, см., например, [37], [23], [60], [95], [73] и в особенности [14]. Исчерпьваюший обзор алгебраических аспектов теории наборов комплексных гиперплоскостей дан в статье С. Юзвинского [90].

В настоящей короткой статье я старался дать элементарное введение в теорию, уделяя особое внимание а) наиболее геометрическим аспектам и мотивировкам этой теории, б) недавним результатам, не отраженным еще в популярных текстах, в) темам, которые традищионно описываются более формальньм и абстрактным образом, чем это можно сделать, г) результатам, имеющим важные приложения и обобщения в знакомых мне областях: дифференциальной топологии, теории особенностей, интегральной геометрии, теории сложности ... .

\section{§2. Основные определения, обозначения и примеры}

Основной объект, рассматриваемый в статье, - это произвольный конечный набор афффинных плоскостей (произвольных, быть может различных, размерностей) в $\mathbb{R}^{N}$. Для краткости такой объект будет называться просто набором плоскостей.

При частичной поддержке NWO (проект 047-008-005). 
Набор плоскостей называется иентральным, если все его плоскости содержат начало координат в $\mathbb{R}^{N}$. В этом случае говорят также, что мы имеем набор подпространств.

Можно определить также комплексные наборы плоскостей или подпространств в $\mathbb{C}^{N}$ : они являются частньм случаем обычных наборов плоскостей в $\mathbb{R}^{2 N}$.

Аналогичным образом определяются наборы плоскостей в $\mathbb{R} \mathbb{P}^{N}$ или в $\mathbb{C P}^{N}$ : они находятся в очевидном взаимно однозначном соответствии с центральными наборами нетривиальных плоскостей в $\mathbb{R}^{N+1}\left(\right.$ соответственно, в $\left.\mathbb{C}^{N+1}\right)$.

Любая аффинная плоскость в $\mathbb{R}^{N}$ определяет комплексную плоскость той же размерности в $\mathbb{C}^{N}$ - свою комплексификацию. Тем самым корректно определена комплексификация любого вещественного набора плоскостей.

Пусть мы имеем набор плоскостей $\mathscr{L}$, состояший из плоскостей $L_{1}, \ldots, L_{m}$. Их объединение $L_{1} \cup \cdots \cup L_{m}$ назьвается носителем набора $\mathscr{L}$ и будет обозначаться через $L$. Для любого набора индексов $I \subset\{1, \ldots, m\}$ положим

$$
L_{I} \equiv \bigcap_{i \in I} L_{i}
$$

Простейший пример набора гиперплоскостей дается координатнымм крестом в $\mathbb{R}^{N}$, определенным уравнением $x_{1} \cdots x_{N}=0$.

Следуюший знаменитьй набор плоскостей - диагональный набор $A(N, 2) \subset \mathbb{C}^{N}$ - состоит из $\left(\begin{array}{c}N \\ 2\end{array}\right)$ гиперплоскостей $V_{i j} \equiv V_{j i},\{i \neq j\} \subset\{1, \ldots, N\}$, заданных уравнениями $x_{i}=x_{j}$. Дополнение к этому набору в $\mathbb{C}^{N}$ можно рассматривать как $N-e$ упорядоченное конфигурационное пространство многообразия $\mathbb{C}^{1}$, т.е. пространство всех упорядоченных наборов из $N$ попарно различных точек в $\mathbb{C}^{1}$.

Более обшим образом для любого $k \in[2, N]$ набор $k$-совпадения $A(N, k)$ состоит из $\left(\begin{array}{c}N \\ k\end{array}\right)$ плоскостей $V\left(i_{1}, \ldots, i_{k}\right), 1 \leqslant i_{1}<\cdots<i_{k} \leqslant N$, заданных условиями $x_{i_{1}}=$ $\cdots=x_{i_{k}}$. Такой набор можно определить как в $\mathbb{R}^{N}$, так и в $\mathbb{C}^{N}$.

Вот еще одно обобщение набора $A(N, 2)$ (см. $[22])$. Рассмотрим любую конечную групу $W$ изометрий евклидова пространства $\mathbb{R}^{N}$, порожденную отражениями в нескольких гиперплоскостях (зеркалах). (Такие группы хорошо известны, см. [21]: неприводимые групшы этого типа образуют четыре бесконечные серии $A_{m}(m \geqslant 1)$, $C_{m}(m \geqslant 2), D_{m}(m \geqslant 4)$ и $I_{2}(p)$ и семь исключительных случаев $G_{2}, F_{4}, H_{3}, H_{4}, E_{6}$, $E_{7}$ и $E_{8}$.) Почти все орбиты действия $W$ в $\mathbb{R}^{N}$ имеют одну и ту же мошность. Объединение нерегулярных орбит меньшей мошности состоит из зеркал, порождаюших группу, и их образов при действии этой группы. Все компоненты дополнения к этому объединению являются симплициальными конусами (камерами Вейля). Действие $W$ в пространстве $\mathbb{R}^{N}$ продолжается очевидным образом до действия в его комплексификации $\mathbb{C}^{N}$. Объединение $D_{W}$ нерегулярных орбит последнего действия состоит из комплексификаций зеркал и их орбит. Оно назьвается диагональю групы $W$.

Пусть, например, зеркала задаются уравнениями $x_{i}=x_{j}, i \neq j$. Тогда группа $W$ изоморфна группе перестановок $S(N)$, и соответствуюший диагональный набор плоскостей совпадает с $A(N, 2)$. Это - случай $A_{N-1}$ из классификации групп, порожденных отражениями.

Набор гиперплоскостей в $\mathbb{R}^{N}$ или в $\mathbb{C}^{N}$, или в $\mathbb{R P}^{N}$, или в $\mathbb{C P}^{N}$ имеет нормальнье пересечения, если для любого подмножества $I \subset\{1, \ldots, m\}$ либо плоскость $L_{I}$ пуста, либо ее коразмерность $N-\operatorname{dim} L_{I}$ равна мощности множества $I$. 
Набор гиперплоскостей в $\mathbb{R}^{N}$ или в $\mathbb{C}^{N}-$ типичныц й (или, эквивалентно, общего положсения), если, будучи дополнен несобственной гиперплоскостью, он становится набором гиперплоскостей с нормальньми пересечениями в $\mathbb{R P}^{N}$ (соответственно, в $\left.\mathbb{C P}^{N}\right)$.

Легко видеть, что для всех типичных наборов из $m$ гиперплоскостей в $\mathbb{C}^{N}$ соответствующие тройки $\left(\mathbb{C P}^{N}, \mathbb{C}^{N}, L\right)$ гомеоморфны. Типичные наборы образуют открытое плотное подмножество в пространстве всех упорядоченных наборов из $m$ гиперплоскостей.

\section{§3. Основной пример: кольцо когомологий группы крашеных кос}

Обозначим открытое многообразие $\mathbb{C}^{N} \backslash A(N, k)$ через $M(N, k)$.

ПРеДЛОЖЕНИЕ 1 (см. [31], [4]). Для любого $N$ многообразие $M(N, 2)$ является пространством типа $K(\pi, 1)$, т.е. все его стариие гомотопические группь $\pi_{2}, \pi_{3}, \ldots$ тривиальньи

Действительно, забьвание последней точки набора из $N$ точек определяет расслоение

$$
M(N, 2) \rightarrow M(N-1,2)
$$

слой которого равен $\mathbb{C}^{1} \backslash\{N-1$ точек $\}$. Предложение 1 доказьвается по индукции с помощью точной гомотопической последовательности этого расслоения.

Группа $\pi_{1}(M(N, 2))$ называется группой крашеных кос с $N$ нитями. Фундаментальная группа аналогичного пространства неупорядоченных наборов назьвается просто группой кос с $N$ нитями, см. [8]. Об алгебраических и гомологических свойствах этих групп см., в частности, [50], [5], [41], [66].

Аналогичное утверждение справедливо для любой группы, порожденной отражениями.

ТеОрема 1 (см. [22], [26]). Для любой конечной группь $W$, действующей отражениями в $\mathbb{R}^{N}$, соответствующее пространство $\mathbb{C}^{N} \backslash D_{W}$ (т.е. оббединение регулярных орбит комплексифицированного действия в $\left.\mathbb{C}^{N}\right)$ является пространством muпа $K(\pi, 1)$.

Эта теорема была доказана Э. Брискорном для групп типа $C_{m}, D_{m}, G_{2}, F_{4}, I_{2}(p)$; П. Делинь доказал ее в обшем виде. Группы $\pi_{1}\left(\mathbb{C}^{N} \backslash D_{W}\right)$ для этих наборов назьваются группами кос Брискорна, см. [22].

Из теоремы 1 следует, что кольца когомологий этих групп изоморфны кольцам когомологий пространств $\mathbb{C}^{N} \backslash D_{W}$. Эти кольца были вычислены в [4] для случая $A_{N-1}$ (т.е. для набора гиперплоскостей $\left.D_{W} \equiv A(N, 2)\right)$ и в [22] для всех остальных групп, порожденных отражениями.

Более того, дополнение $M_{\mathbb{R}}(N, 3)$ к вещественному набору 3-совпадений также является пространством типа $K(\pi, 1)$ для любого $N$ : это утверждение было высказано А. Бъёрнером в качестве гипотезы и доказано М. Ховановым [47].

Вычисление кольца когомологий в арнольдовском случае $M(N, 2)$ вновь основано на расслоении (2).

ПРЕДЛОЖЕНИЕ 2 (см. [4]). Кольио $H^{*}(M(N, 2))$ не имеет кручения и изоморфно тензорному произведению $H^{*}\left(\varphi_{N-1}\right) \otimes H^{*}\left(\varphi_{N-2}\right) \otimes \cdots \otimes H^{*}\left(\varphi_{1}\right)$, где $\varphi_{i}-$ 
букет і окружностей. В частности, полином Пуанкаре кольца $H^{*}(M(N, 2))$ равен $(1+t)(1+2 t) \cdots(1+(N-1) t)$.

Действительно, легко видеть, что расслоение (2) гомологически просто, т.е. фундаментальная група базы тривиально действует на гомологиях слоя (гомотопически эквивалентного $\left.\varphi_{N-1}\right)$. Спектральная последовательность этого расслоения стабилизируется в члене $E_{2}$, и мы получаем кольцевой изоморфизм $H^{*}(M(N, 2)) \simeq H^{*}(M(N-1,2)) \otimes H^{*}\left(\varphi_{N-1}\right)$.

Более симметричное описание этого кольца, также полученное в [4], состоит в следующем. Для каждой плоскости $V_{j k}$ нашего набора обозначим через $\omega_{j k}$ логарифмическую форму с особенностью на этой плоскости: $\omega_{j k} \equiv \frac{1}{2 \pi i} \frac{d z_{j}-d z_{k}}{z_{j}-z_{k}}$. Интеграл этой формы по любому замкнутому пути в $M(N, 2)$ равен индексу зацепления этого пути с плоскостью $V_{j k}$.

ПреДЛОЖенИЕ 3. Для любых трех различных индексов $i, j, k \in[1, N]$ равенство

$$
\omega_{i j} \wedge \omega_{j k}+\omega_{j k} \wedge \omega_{k i}+\omega_{k i} \wedge \omega_{i j}=0
$$

выполняется тождественно в $M(N, 2)$. В частности, выполнено аналогичное равенство для соответствующих классов когомологий в кольце $H^{*}(M(N, 2))$ : если $\Omega_{j k}-$ когомологический класс формы $\omega_{j k}$, то

$$
\Omega_{i j} \smile \Omega_{j k}+\Omega_{j k} \smile \Omega_{k i}+\Omega_{k i} \smile \Omega_{i j}=0 .
$$

Алгебра челочисленных когомологий $H^{*}(M(N, 2))$ канонически изоморфна факторалгебре внешней алгебры, формально порожденной $\left(\begin{array}{c}N \\ 2\end{array}\right)$ әлементами $\Omega_{j k}$, по идеалу, мультипликативно порожденному левыми частями всевозможных выражений (4) с произвольнымми $i, j$ и $k$.

\section{$\S 4$. Кольцо Орлика-Соломона и когомологии дополнения к набору комплексных гиперплоскостей}

Аналог предложения 3 имеет место и для произвольного центрального набора комплексных гиперплоскостей.

Пусть $L=\left\{L_{1}, \ldots, L_{m}\right\}-$ такой набор в $\mathbb{C}^{N}$, плоскости $L_{i}$ которого задаются линейными уравнениями $f_{i}=0$. Набор индексов $I \subset\{1, \ldots, m\}$ называется зависимым, если коразмерность плоскости $L_{I}$ меньше, чем ожидаемое значение $|I|$ (т.е. соответствуюшие уравнения $f_{i}$ линейно зависимы). Для любого зависимого множества $I=\left\{i_{1}<\cdots<i_{k}\right\}$ обозначим через $\rho(I)$ рациональную дифференциальную $(k-1)$-форму

$$
\sum_{j=1}^{k}(-1)^{j} \frac{d f_{i_{1}}}{f_{i_{1}}} \wedge \cdots \wedge \frac{\widehat{d f_{i_{j}}}}{f_{i_{j}}} \wedge \cdots \wedge \frac{d f_{i_{k}}}{f_{i_{k}}}
$$

Легко видеть, что эта форма равна нулю в $\mathbb{C}^{N} \backslash L$. 
ТЕОРема 2 (см. [59]). Для любого центрального набора комплексных гиперплоскостей $\mathscr{L}$ в $\mathbb{C}^{N}$ кольцо иелочисленных когомологий $H^{*}\left(\mathbb{C}^{N} \backslash L\right)$ канонически изоморфно факторалгебре внешней алгебрь с образующими $\alpha_{j}$, соответствующими гиперплоскостям нашего набора $\mathscr{L}$, по идеалу, порожденному всевозможными әлементами

$$
\sum_{j=1}^{k}(-1)^{j} \alpha_{i_{1}} \smile \cdots \smile \widehat{\alpha_{i_{j}}} \smile \cdots \smile \alpha_{i_{k}},
$$

соответствующими зависимьм наборам $I=\left(i_{1}, \ldots, i_{k}\right)$. Более того, этот же идеал порождается выражениями (6), соответствующими только минимальным зависимым маборам I.

Пример. Для набора $A(N, 2)$ минимальные зависимые наборы - это наборы плоскостей вида $V_{i_{1} i_{2}}, V_{i_{2} i_{3}}, \ldots, V_{i_{q-1}} i_{q}, V_{i_{q} i_{1}}$, где $\left\{i_{1}, \ldots, i_{q}\right\} \subset\{1, \ldots, N\}$ - произвольньй набор индексов, $q \geqslant 3$. Легко видеть, что любая форма (6), заданная таким набором, принадлежит идеалу, порожденному аналогичными формами с $q=3$.

Основной шаг к теореме 2 был сделан в [22].

СлЕДСТВИе 1 (см. [22]). Группа $H^{*}\left(\mathbb{C}^{N} \backslash L\right)$ не имеет кручения.

Случай нецентрального набора гиперплоскостей в $\mathbb{C}^{N}$ легко сводится к случаю центрального набора в $\mathbb{C}^{N+1}$.

\section{§. В какой степени топология дополнения определяется размерностными данными: сводка результатов}

Пусть $\mathscr{L}$ - произвольный аффинный набор из $m$ плоскостей в $\mathbb{R}^{N}$. Допустим, что для любого $I \subset\{1, \ldots, m\}$ мы знаем размерность плоскости $L_{I}$ (а также пуста эта плоскость или нет). Что тогда можно сказать о топологии пространства $\mathbb{R}^{N} \backslash L$ ? Верно ли, что тот или иной топологический инвариант этого пространства однозначно определяется этими данньми?

Для групп гомологий и когомологий $\mathbb{R}^{N} \backslash L$ ответ на последний вопрос положителен, см. [40] и $\S 6$ ниже.

Для стабильного гомотопического типа ответ также положителен, см. [76], [96] и $\S 8$ ниже.

Для мультипликативной структуры в когомологиях, ответы таковы.

А. В наиболее общей ситуации ответ отрицателен, см. [94], [95].

В. Для комплексных наборов плоскостей произвольных размерностей ответ положителен. Для наборов гиперплоскостей это следует из предыдущей теоремы Орлика-Соломона. В случае произвольного комплексного набора это было доказано в [24] для рациональных когомологий. Затем С. Юзвинский [91] предложил явную формулу для этого умножения в рациональных когомологиях, и наконец было доказано [28], [29], что эта же формула описывает умножение и в кольце целочисленных когомологий, см. $\S 11$ ниже.

С. Имеется более обший класс вешественных наборов плоскостей, для которых значительная часть мультипликативной структуры определяется размерностньми данньми.

ОПреДЕЛЕнИЕ. Набор плоскостей $\mathscr{L}$ в $\mathbb{R}^{N}$ назьвается $\geqslant 2$-набором, если для любых двух его инцидентных плоскостей $L_{I} \varsubsetneqq L_{J}$ вьполняется $\operatorname{dim} J-\operatorname{dim} I \geqslant 2$. 
Например, любой комплексньй набор удовлетворяет этому условию.

Для таких наборов в [28], [29] была доказана явная формула умножения в когомологиях. В частности, из нее вытекает следующее предложение.

Пусть две плоскости $L_{I}, L_{J}$ нашего набора трансверсальны, т.е.

$$
N-\operatorname{dim}\left(L_{I} \cap L_{J}\right)=\left(N-\operatorname{dim} L_{I}\right)+\left(N-\operatorname{dim} L_{J}\right) .
$$

Зафиксируем также ориентацию в $\mathbb{R}^{N}$. Тогда любой выбор ориентаций плоскостей $L_{I}, L_{J}$ стандартным образом задает ориентацию плоскости $L_{I} \cap L_{J} \equiv L_{I \cup J}$. Если фиксированы ориентации всех плоскостей $L_{I}$ нашего набора, то для каждой упорядоченной пары трансверсальных плоскостей $L_{I}, L_{J}$ мы получаем знак + или -, зависяший от того, совпадает ли фиксированная ориентация плоскости $L_{I \cup J}$ с ориентацией, индуцированной фиксированными ориентациями плоскостей $L_{I}$ и $L_{J}$.

ПРЕДЛОЖЕНИЕ 4. Пусть мы имеем два $\geqslant 2$-набора плоскостей $\mathscr{L}, \mathscr{L}^{\prime}$ в $\mathbb{R}^{N}$ таких, что $\operatorname{dim} L_{I}=\operatorname{dim} L_{I}^{\prime}$ для всякого $I$, и имеются системь ориентаций всех этих плоскостей такие, что для любой парь мультииндексов I, J, удовлетворяющих условию (7), соответствующие знаки совпадают. Тогда кольца когомологий пространств $\mathbb{R}^{N} \backslash L, \mathbb{R}^{N} \backslash L^{\prime}$ изоморфньл.

Конечно, комплексные наборы с одинаковыми размерностньми данными удовлетворяют условиям этого предложения: если мы выберем комплексные ориентации всех плоскостей, то все знаки будут равны + .

D. C другой стороны, условие на согласование ориентаций нельзя отбросить: соответствуюший контрпример см. в [94].

$\mathbf{E . ~ В с е ~ ж е ~ ч т о - т о ~ м о ж н о ~ с к а з а т ь ~ д а ж е ~ в ~ н а и б о л е е ~ о б щ е м ~ с л у ч а е ~ п р о и з в о л ь н о г о ~ в е - ~}$ щественного набора аффинных плоскостей. Группа $H^{*}\left(\mathbb{R}^{N} \backslash L\right)$ всегда обладает естественной фильтрацией, см. $\S \S 6,8$ ниже. Соответствующее градуированное кольио однозначно определяется размерностными данными и системой знаков как в п. C выше. Его описание также вытекает из результатов [28], [29], см. §11.

Наиболее хрупким инвариантом оказьвается фундаментальная группа дополнения к набору плоскостей.

ТеОрема 3 (см. [64]). Существуют два набора комплексных прямых в $\mathbb{C}^{2}$ с одинаковыми размерностными даннымми (т.е. наборами прямых, проходящих через одну точку), но с неизоморфными фундаментальными группами дополнений до их носителей.

Наконец, если мы рассмотрим топологию не дополнения к носителю $L$, а самого этого носителя или его одноточечной компактификации $\bar{L}$, то размерностная информация оказьвается очень сильной.

Tеорема 4 (см. §8). Гомотопические типь топологических пространств L и $\bar{L}$ полностью определяются размерностными данными.

\section{§ 6. Порядковый комплекс частично упорядоченного множества. Формула Горески-Макферсона}

Для произвольного аффинного набора вешественных плоскостей группа когомологий его дополнения была вычислена М. Горески и Р. Макферсоном в качестве яркого приложения их стратифицированной теории Морса [40]. 
В нашем частном случае эта теория работает следуюшим образом. Зафиксируем типичную точку $X_{0} \in \mathbb{R}^{N} \backslash L$ и положительную квадратичную функцию $f: \mathbb{R}^{N} \rightarrow \mathbb{R}$ с центром в этой точке (т.е. равную $x_{1}^{2}+\cdots+x_{N}^{2}$ в некоторых афффинных координатах с центром в $X_{0}$ ). Критические значения функции $f$ - это критические значения ее ограничений на всевозможные непустые плоскости $L_{I}$ нашего набора. Если $X_{0}$ и $f$ действительно находятся в обшем положении по отношению к $\mathscr{L}$, то все эти значения, соответствуюшие разным плоскостям $L_{I}$, различны. Далее, для любого положительного $t$ рассмотрим шар $B_{t} \equiv\{x: f(x) \leqslant t\}$ в $\mathbb{R}^{N}$ и многообразие меньших значений $\Lambda(t) \equiv B_{t} \backslash L$. Если отрезок $[a, b]$ не содержит критических значений $f$, то $\Lambda(a)$ и $\Lambda(b)$ гомотопически эквивалентны (и даже гомеоморфны). Если $t$ достаточно мало, то $\Lambda(t)$ - шарик; если $t$ достаточно велико, то $\Lambda(t)$ является деформационньм ретрактом исследуемого пространства $\mathbb{R}^{N} \backslash L$. Следовательно, $\mathbb{R}^{N} \backslash L$ строится из топологически тривиального пространства при помощи конечной последовательности локальных перестроек, соответствуюших всевозможным критическим значениям $f$.

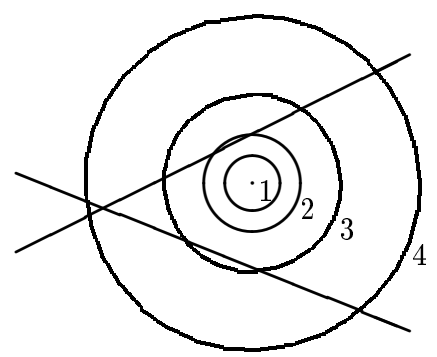

Рис. 1. Эволюция множеств меньших значений

Рассмотрим, например, набор прямых в $\mathbb{R}^{2}$, изображенный на рис. 1 , и его комплексификацию $\mathscr{L}$ в $\mathbb{C}^{2}$. Имеется четыре сушественно различных некритических значения $t_{1}<t_{2}<t_{3}<t_{4}$; пересечения соответствуюших шаров $B_{t_{i}}$ с $\mathbb{R}^{2}$ изображены на рис. 1 . Пусть $\Lambda_{i} \equiv \Lambda\left(t_{i}\right)$ - соответствуюшие многообразия нашего индуктивного процесса.

Многообразие $\Lambda_{1}$ топологически тривиально. Переходы от $\Lambda_{1}$ к $\Lambda_{2}$ и от $\Lambda_{2}$ к $\Lambda_{3}$ гомотопически эквивалентны приклеиванию 1 -мерных клеток, так что $\Lambda_{3}$ гомотопически эквивалентно букету двух окружностей. Наконец, переход от $\Lambda_{3}$ к $\Lambda_{4}$ эквивалентен приклеиванию 2-мерной клетки. И действительно, легко видеть, что полученное многообразие $\Lambda_{4}$ гомотопически эквивалентно двумерному тору.

Вообще, для любого набора плоскостей индуктивное вычисление групп гомологий пространства $\mathbb{R}^{N} \backslash L$ включает локальные проблемы следуюшего типа. Допустим, что значения $a, b$ некритичны и отрезок $[a, b]$ содержит ровно одно критическое значение функции $f$, а именно, критическое значение ограничения $f$ на некоторую плоскость $L_{I}$. Топологические типы многообразий $\Lambda_{a}, \Lambda_{b}$ различаются перестройкой, локализованной в малой окрестности соответствующей критической точки на $L_{I}$. Как связьвает она группы когомологий этих многообразий?

Эта задача решена в [40] в комбинаторных терминах нашего набора плоскостей. Ответ формулируется в терминах порядкового комплекса частично упорядоченного множества.

ОПРЕДЕЛЕНИЕ. Пусть дано частично упорядоченное множество $(A,<)$, тогда соответствуюший порядковый комплекс $\Upsilon(A)$ - это симплициальный комплекс, вершинами которого являются точки множества $A$, а симплексы натянуты на всевозможные последовательности таких точек, монотонные относительно частичного порядка. 
Любой набор плоскостей $\mathscr{L}=\left\{L_{1}, \ldots, L_{m}\right\}$ определяет частично упорядоченное множество всех его непустых плоскостей $L_{I}, I \subset\{1, \ldots, m\}$, а следовательно, и порядковый комплекс $\Upsilon(\mathscr{L})$.

Например, для трех наборов прямых, изображенных в нижнем ряду рис. 2 , соответствующие порядковые комплексы показаны на рис. 3. Для диагонального набора $A(4,2)($ см. $\S 2)$ порядковьй комплекс изображен на рис. 4.

С каждьм множеством $I$ таким, что $L_{I} \neq \varnothing$, связан порядковый подкомплекс $\Upsilon(I) \subset \Upsilon(\mathscr{L})$ : это объединение всех симплексов в $\Upsilon(\mathscr{L})$ таких, что все их вершины соответствуют плоскостям $L_{J}$, содержашим $L_{I}$. Любой такой подкомплекс $\Upsilon(I)$ стягиваем: действительно, все его максимальные симплексы имеют общую вершину, соответствуюшую само́й плоскости $\Upsilon(I)$. Обозначим через $\partial \Upsilon(I)$ линк этого подкомплекса, т.е. объединение всех его симплексов, не содержаших его максимальную вершину $\{\Upsilon(I)\}$.

Tеорема 5 (см. [40]). Допустим, что отрезок $[a, b]$ содержит ровно одно критическое значение $f$; пусть $L_{I}$ - соответствующая плоскость. Тогда

1) $H^{i}(\Lambda(b), \Lambda(a)) \simeq H_{N-i-\operatorname{dim} L_{I}-1}(\Upsilon(I), \partial \Upsilon(I))$;

2) точная гомологическая последовательность пары $(\Lambda(b), \Lambda(a))$ расщепляeтся, m.е. $\widetilde{H}^{*}(\Lambda(b)) \simeq H^{*}(\Lambda(b), \Lambda(a)) \oplus \widetilde{H}^{*}(\Lambda(a)) ;$ здесь $\widetilde{H}^{*}$ обозначает группу когомологий, приведенную по модулю точки.

СЛЕДСТВИЕ 2 (см. [40]). Для произвольного аффинного набора плоскостей $\mathscr{L}$ $\boldsymbol{\theta} \mathbb{R}^{N}$

$$
\widetilde{H}^{i}\left(\mathbb{R}^{N} \backslash L\right) \simeq \bigoplus_{I} H_{N-i-\operatorname{dim} L_{I}-1}(\Upsilon(I), \partial \Upsilon(I))
$$

суммирование - по всем непустым плоскостям $L_{I}$ набора.

В $\S 8$ мы объясним эту формулу и докажем ее гомотопический вариант.
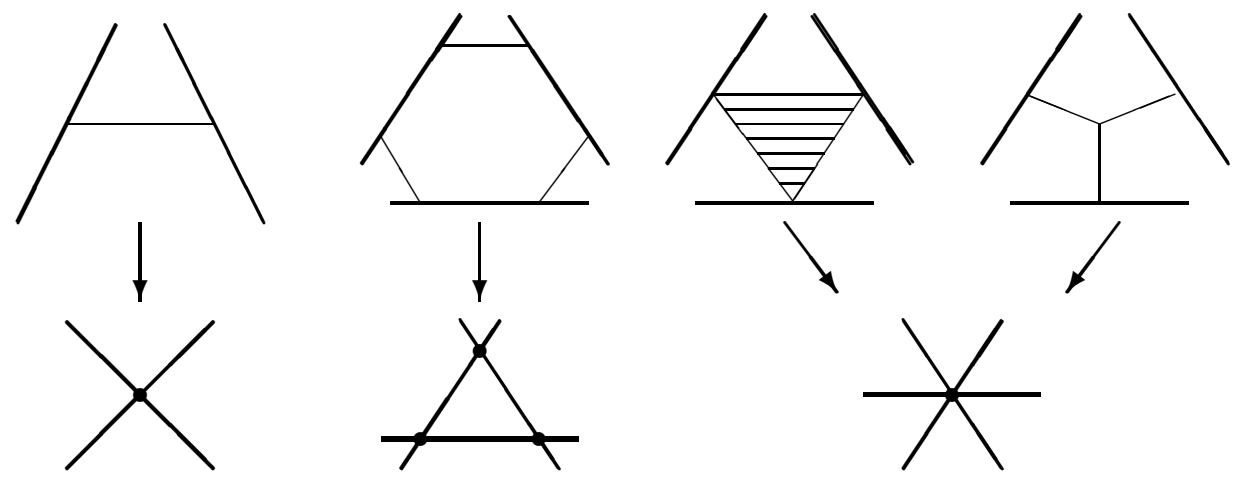

Рис. 2. Симплициальные разрешения наборов прямых

ПримеР. Допустим, что мы имеем набор комплексных гиперплоскостей с нормальными пересечениями в $\mathbb{C}^{N}$. Тогда группа $H^{r}\left(\mathbb{C}^{N} \backslash L\right)$ изоморфна $\mathbb{Z}^{\lambda(r)}$, где $\lambda(r)$ - число наборов индексов $I=\left\{i_{1}, \ldots, i_{r}\right\}$ мошности $r$ таких, что $L_{I} \neq \varnothing$. Действительно, для любого такого множества $I$ соответствуюший порядковый подкомплекс $\Upsilon(I)$ совпадает с первым барищентрическим подразбиением $(r-1)$-мерного симплекса, линк его совпадает с границей этого симплекса, группа $H_{*}(\Upsilon(I), \partial \Upsilon(I))$ имеет единственньй нетривиальный член $\mathbb{Z}$ в размерности $r-1$, и $\operatorname{dim}_{\mathbb{R}} L_{I}=2 N-2 r$. В частности, для 


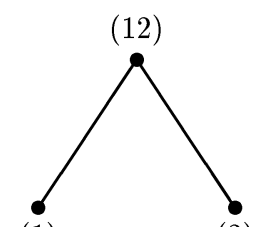

(1)
$(2)$

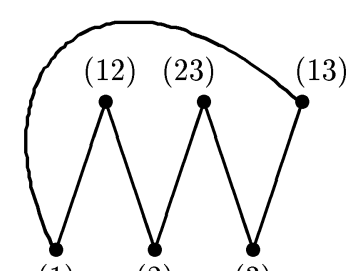

(1)

(2)

(3)

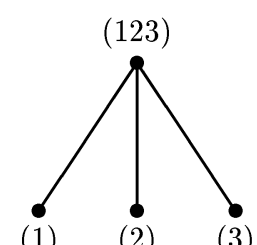

Рис. 3. Порядковые комплексы для наборов прямшхх

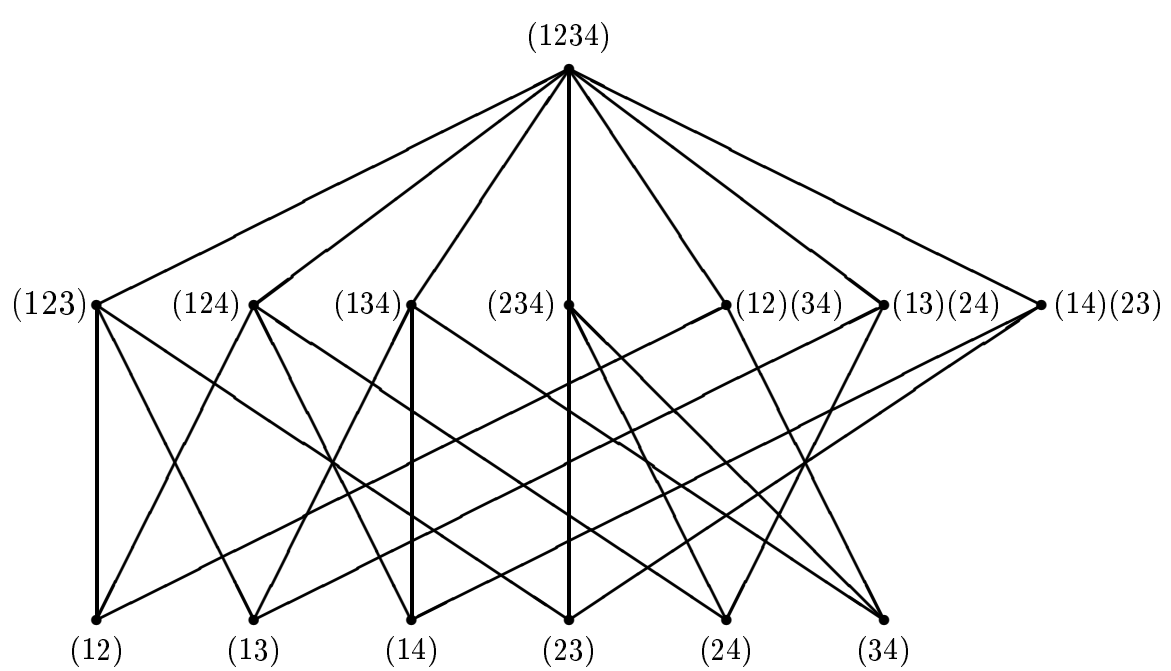

Рис. 4. Частично упорядоченное множество и порядковый комплекс для диагонального набора $A(4,2)$

типичного набора из $m$ гиперплоскостей в $\mathbb{C}^{N}$ мы имеем $\left.H^{r}\left(\mathbb{C}^{N} \backslash L\right) \sim \mathbb{Z}^{(m} r\right)$ для любого $r=0,1, \ldots, N$. В последнем случае верно более сильное утверждение: пространство $\mathbb{C}^{N} \backslash L$ гомотопически эквивалентно $N$-мерному остову стандартного клеточного разложения $m$-мерного тора. Действительно, рассмотрим универсальный набор из $m$ гиперплоскостей $\dagger^{m}$, т.е. координатный крест в $\mathbb{C}^{m}$. Сушествует аффинное вложение $\varphi: \mathbb{C}^{N} \rightarrow \mathbb{C}^{m}$ такое, что плоскости нашего типичного набора в $\mathbb{C}^{N}$ являются прообразами пересечений $\varphi\left(\mathbb{C}^{N}\right)$ с координатными плоскостями в $\mathbb{C}^{m}$. Группу когомологий пространства $\mathbb{C}^{m} \backslash \dagger^{m} \sim T^{m}$ можно вьчислять, как вьше, с помошью произвольной типичной вещественной квадратичной функции $f: \mathbb{C}^{m} \rightarrow \mathbb{R}$. Она имеет ровно $2^{m}-1$ критических значений, соответствуюших всевозможным плоскостям $\dagger_{I}^{m}$, а перестройки, соответствуюшие переходам через эти критические значения, гомотопически эквивалентны добавлению клеток стандартного клеточного разложения тора. Но мы можем выбрать нашу функцию $f$ так, чтобы ее нулевая точка лежала на вложенной плоскости $\varphi\left(\mathbb{C}^{N}\right) \subset \mathbb{C}^{m}$, и чтобы она росла очень медленно вдоль $\varphi\left(\mathbb{C}^{N}\right)$ и очень быстро в направлениях, трансверсальных к ней. "Шары" $\{x: f(x) \leqslant t\}$ будут вьплядеть как блины, размазанные вдоль $\varphi\left(\mathbb{C}^{N}\right)$. Для некоторого $t$ такой шар будет пересекать все плоскости $L_{I}$ с $|I| \leqslant N$, но не пересекать плоскостей меньшей размерности. Соответствующее многообразие $\Lambda(t)$ гомотопически эквивалентно $\mathbb{C}^{N} \backslash L$, с другой стороны, оно гомотопически эквивалентно $N$-мерному остову тора $T^{m} \sim \mathbb{C}^{m} \backslash \dagger^{m}$. 
Изоморфизм (8), как он возникает из его доказательства в [40], неканоничен: его реализация зависит от некоторого произвола. Однако он позволяет определить важную возрастаюшую фильтрацию в групе $H^{*}\left(\mathbb{R}^{N} \backslash L\right)$ : для произвольной реализации этого изоморфизма член $F_{i}$ этой фильтрации соответствует сумме членов $H_{*}(\Upsilon(I), \partial \Upsilon(I))$ по всем плоскостям $L_{I}$ коразмерностей $\leqslant i$. Это определение уже не зависит от произвола, см. $\S \S 8,10$.

\section{$\S$ 7. Симплициальные разрешения и формула включений-исключений. Спектральная последовательность Майера-Вьеториса и ее модификации}

Симплициальные разрешения являются далеким обобщением комбинаторной формулы включений-исключений. Они позволяют эффективно изучать топологию пространств, представленных как объединения нескольких подпространств, пересечения которых (в том числе кратные) устроены гораздо проще, чем их симметрические разности.

Вначале продемонстрируем этот метод в простейшей дискретной ситуации. Допустим, что конечное множество $S$ представлено как объединение конечного набора конечных подмножеств $S_{i}, i=1, \ldots, m$, и нам требуется найти мошность $S$. Для этого построим симплициальное разрешение $S$. Вначале мы возьмем все множества $S_{i}$ по отдельности. Если два множества $S_{i}, S_{j}$ имеют общую точку, то проведем отрезок, соединяющий соответствуюшие точки в разделенных экземплярах $S_{i}$ и $S_{j}$. Если некоторая точка принадлежит тройному пересечению $S_{i} \cap S_{j} \cap S_{k}$, то мы получаем три разделенные точки, соединенные тремя отрезками. На следующем шаге мы добавляем “внутреннюю часть" этого треугольника, затем строим тетраэдры над четырехкратными точками и т. д. Полученньй комплекс $S^{\prime}$ гомотопически эквивалентен исходному множеству $S$ : каждой точке из $S$ соответствует симплекс в $S^{\prime}$. В частности, $S$ и $S^{\prime}$ имеют одинаковые эйлеровы характеристики. Но эйлерова характеристика конечного множества $S$ - это его мошность, а таковая множества $S^{\prime}$ - это число его вершин (т.е. точек во всех множествах $S_{i}$, взятых по отдельности) минус число ребер (т.е. сумма мошностей всех множеств $S_{i} \cap S_{j}$ по всем парам $\left.(i \neq j) \subset\{1, \ldots, r\}\right)$ плюс число треугольников и т. д. Результат есть не что иное, как формула включений-исключений.

Этот же метод работает в "непрерьвном" случае, скажем, если множество $S=$ $\bigcup S_{i}$ является $C W$-комплексом, а все множества $S_{i}$ и все их пересечения $S_{I}$ являются его клеточными подкомплексами. Именно, мы рассматриваем $(m-1)$-мерный симплекс $\Delta$, вершины которого находятся во взаимно однозначном соответствии с индексами $1, \ldots, m$, нумеруюшими наши множества $S_{i}$. Симплициальное разрешение можно построить как подмножество прямого произведения $\Delta \times S$. Для любого набора индексов $I$ мы берем симплекс $\Delta(I)$, вершины которого соответствуют точкам множества $I$. Симплициальное разрешение $S^{\prime}$ определяется как объединение всех произведений $\Delta(I) \times S_{I}$ по всем множествам $I$. Очевидное проектирование $\Delta \times S \rightarrow S$ индуцирует отображение $S^{\prime} \rightarrow S$. Легко видеть, что это отображение собственно и является гомотопической эквивалентностью. Часто случается, что изучать это пространство $S^{\prime}$ гораздо проще, чем исходное пространство $S$.

ПримеР. Если имеется только два множества $S_{1}, S_{2}$ и $S=S_{1} \cup S_{2}$, то мыполучаем точную последовательность Майера-Вьеториса

$$
\cdots \rightarrow H_{i}\left(S_{1} \sqcup S_{2}\right) \rightarrow H_{i}(S) \rightarrow H_{i-1}\left(S_{1} \cap S_{2}\right) \rightarrow H_{i-1}\left(S_{1} \sqcup S_{2}\right) \rightarrow \cdots
$$


Действительно, соответствующий симплекс $\Delta$ - это отрезок $[1,2]$. Несвязное объединение $S_{1} \sqcup S_{2}$ можно реализовать как подмножество $\left(\{1\} \times S_{1}\right) \cup\left(\{2\} \times S_{2}\right) \subset[1,2] \times$ $\left(S_{1} \cup S_{2}\right)$, и мы можем рассмотреть точную последовательность пары $\left(S^{\prime},\left(\{1\} \times S_{1}\right) \cup\right.$ $\left.\left(\{2\} \times S_{2}\right)\right)$.

Более обшим образом, для любого количества $m$ множеств $S_{i}$ разрешенньй комплекс $S^{\prime}$ также имеет стандартную фильтрацию $\varphi_{1} \subset \cdots \subset \varphi_{m}$ : ее множество $\varphi_{k}-$ это объединение по $l=1, \ldots, k$ всевозможных множеств $\Delta(I) \times S_{I}$ с $l$-элементными подмножествами $I \in\{1, \ldots, m\}$. Соответствующая спектральная последовательность, вычисляющая гомологии пространства $S^{\prime} \sim S$, назьвается спектральной последовательностью Майера-Вьеториса составного множества $S=\bigcup S_{i}$. Она бьвает полезна в некоторых топологических задачах, однако при изучении наборов плоскостей она довольно бесполезна: в этом случае нужно рассматривать другую фильтрацию на пространстве разрешения, см. следуюший параграф.

\section{$\S 8$. Гомотопический тип объединения аффинных плоскостей и стабильный гомотопический тип его дополнения}

Пусть наши пространства $S_{i}$ - это аффинные плоскости в $\mathbb{R}^{N}$, составляющие набор $\mathscr{L}$. В нижней части рис. 2 изображены три примера наборов прямых в $\mathbb{R}^{N}$, в верхней части указаны их симплициальные разрешения. Над правой картинкой мы имеем два разных разрешения. Левое из них построено точно как ранее: прообраз центральной точки - это весь симплекс $\Delta$, вершины которого соответствуют всем прямым $L_{i}$.

В обшем случае обозначим через $\Delta(\mathscr{L})$ объединение всех граней $\Delta(I) \subset \Delta$ таких, что плоскость $L_{I}$ непуста. Симплициальное разрешение набора плоскостей $\mathscr{L}$, построенное выше, является подмножеством в $\Delta(\mathscr{L}) \times L$. Другое, более экономное разрешение строится следуюшим образом. Вместо комплекса $\Delta(\mathscr{L}) \subset \Delta$ мы можем рассмотреть порядковый комплекс $\Upsilon(\mathscr{L})$ и определить симплициальное разрешение как подпространство в $\Upsilon(\mathscr{L}) \times L$ : именно, как объединение всех произведений $\Upsilon(I) \times L_{I}$ по всем непустым плоскостям $L_{I}$ нашего набора. Порядковые комплексы трех наборов с рис. 2 показаны на рис. 3 .

Для двух левых наборов с рис. 2 обе конструкции дают одно и то же пространство, но для правого набора вторая конструкция дает другое пространство, см. самую правую верхнюю картинку.

Эти две конструкции гомотопически эквивалентны. Действительно, порядковый комплекс $\Upsilon(\mathscr{L})$ можно отождествить с некоторьм подкомплексом первого барицентрического подразбиения комплекса $\Delta(\mathscr{L})$. При этом вершина $\left\{L_{I}\right\}$ комплекса $\Upsilon(\mathscr{L})$ переходит в центр симплекса из $\Delta(\mathscr{L})$, натянутого на все вершины $\left\{L_{i}\right\}$ такие, что $L_{i} \supset L_{I}$. Это отождествление определяет вложение второй конструкции в первую. Обратно, для любого набора индексов $I$ с непустым $L_{I}$ мы можем отобразить центр симплекса $\Delta(I)$ в вершину $\left\{L_{I}\right\} \in \Upsilon(\mathscr{L}) \subset \Delta(\mathscr{L})$; продолжая это отображение по линейности, мы получим отображение, гомотопически обратное к нашему вложению. Мы будем назьвать эти две конструкции симплициальных разрешений, использующие комплексы $\Delta(\mathscr{L})$ и $\Upsilon(\mathscr{L})$, соответственно наивным. и әкономнылм симплициальными разрешениями.

Почти все последуюшие рассуждения в этом параграфе годятся одинаково для обеих конструкщий. В частности, верно следующее утверждение (см. предложение 5). Допустим, что $L_{I} \neq \varnothing$. Назовем грань $\Delta(J)$ симплекса $\Delta(I)$ маргинальной, если соответствуюшая плоскость $L_{J}$ строго больше, чем $L_{I}$. Обозначим через $\partial \Delta(I)$ подкомплекс в $\Delta(I)$, состояший из всех маргинальных граней. 
ПРЕДЛОЖЕНИЕ 5 .

1. Имеется гомотопическая әквивалентность $\Delta(\mathscr{L}) \sim \Upsilon(\mathscr{L})$.

2. Для любой непустой плоскости $L_{I}$ пары $(\Delta(I), \partial \Delta(I))$ u $(\Upsilon(I), \partial \Upsilon(I))$ гомотопически әквивалентнь.

В частности, формулу Горески-Макферсона (8) можно переписать в следующем виде:

$$
\widetilde{H}^{i}\left(\mathbb{R}^{N} \backslash L\right) \simeq \bigoplus_{I} H_{N-i-\operatorname{dim} L_{I}-1}(\Delta(I), \partial \Delta(I)) .
$$

Обозначим через $L^{\prime}$ пространство разрешения нашего набора плоскостей с носителем $L$, а его одноточечную компактификацию через $\bar{L}^{\prime}$. С ледуюший хорошо известньй факт, базисньй для всей теории симплициальных разрешений, легко вытекает из леммы Борсука, см., например, [84] (к сожалению, я не знаю самой ранней ссылки).

ПРЕДЛОЖЕНИЕ 6. Очевидная проекиия $\pi: L^{\prime} \rightarrow L$ является собственным отображением и гомотопической эквивалентностью. Ее продолэение до отображения одноточечных компактификачий, $\bar{\pi}: \bar{L}^{\prime} \rightarrow \bar{L}$, также является гомотопической әквивалентностью.

ТЕОРема 6. Для любого конечного набора аффинных плоскостей с носителем L имеются гомотопические әквивалентности

$$
\begin{gathered}
L^{\prime} \sim L \sim \Upsilon(\mathscr{L}) \sim \Delta(\mathscr{L}) \\
\bar{L}^{\prime} \sim \bar{L} \sim \bigvee_{I} \Sigma^{\operatorname{dim} L_{I}}(\Upsilon(I) / \partial \Upsilon(I)) \sim \bigvee_{I} \Sigma^{\operatorname{dim} L_{I}}(\Delta(I) / \partial \Delta(I)),
\end{gathered}
$$

где $\Sigma^{k}$ означает k-кратную надстройку; букеть в (12) берутся по всем непустылм плоскостям $L_{I}$ нашего набора.

Средняя эквивалентность в (11) была найдена в [40], см. также [19].

Средняя эквивалентность $\bar{L} \sim \bigvee_{I} \Sigma^{\operatorname{dim} L_{I}}(\Upsilon(I) / \partial \Upsilon(I))$ в (12) была доказанав [96]; одновременно была доказана сквозная эквивалентность $\bar{L}^{\prime} \sim \bigvee_{I} \Sigma^{\operatorname{dim} L_{I}}(\Delta(I) / \partial \Delta(I))$ этого же равенства, см. [76]. В силу предложений 5 и 6 эти два соотношения, участвующие в формуле (12), эквивалентны друг другу и всей этой формуле.

Другие утверждения этого типа см. в [58], [45].

СлЕДСтВИЕ 3. Формула Горески-Макферсона (8), (10).

Действительно, эта формула следует из (12) и двойственности Александера

$$
\widetilde{H}^{i}\left(\mathbb{R}^{N} \backslash L\right) \simeq \bar{H}_{N-i-1}(L),
$$

где $\bar{H}_{*}$ - это группа гомологий Бореля-Mура, т.е. группа гомологий одноточечной компактификации, приведенная по модулю добавленной точки; ср. [5]. (Эквивалентное определение: группа гомологий Бореля-Мура - это группа гомологий комплекса локально конечных сингулярных цепей в $X$.)

Пространство разрешения $L^{\prime}$ обладает очень полезной фильтрацией: ее член $F_{i}(L)$ определяется как объединение всех произведений $\Delta(I) \times L_{I}$ (соответственно, $\left.\Upsilon(I) \times L_{I}\right)$ по всем непустым плоскостям $L_{I}$ коразмерности $\leqslant i$. В частности, $L^{\prime}=F_{N}(L)$. Эта фильтрация продолжается до фильтрациина $\bar{L}^{\prime}$ : член $\bar{F}_{0}$ последнейэто добавленная точка, а $\bar{F}_{i}, i \geqslant 1,-$ это замькание $F_{i}$. Соответствующая фильтрация в гомологиях $\bar{L}^{\prime} \sim \bar{L}$ двойственна по Александеру фильтрации, упоминавшейся в конце $\S 6$. 
СлЕДСТВИЕ 4. Стабильный гомотопический тип дополнения $к$ произвольному набору аффинных плоскостей $\mathscr{L}$ определяется размерностями всех его плоскостей $L_{I}$, в частности, то же верно для всех экстраординарных групп гомологий и когомологий.

Это следствие основано на следующем понятии.

ОПРЕДЕЛЕНИЕ. Два топологических пространства, имеющих гомотопические типы $C W$-комплексов, взаимно двойственнь по Спеньеру-Уайтхедy, если они гомотопически эквивалентны двум дополнительным подмножествам $X$ и $Y \equiv S^{N} \backslash X$ некоторой сфреры $S^{N}$.

Групш гомологий и когомологий таких пространств связаны двойственностью Александера.

Например, наши пространства $\bar{L}$ и $\mathbb{R}^{N} \backslash L$ двойственны по Спеньеру-Уайтхеду.

Важньй факт (см., например, [89]) состоит в том, что двойственность СпеньераУайтхеда определяет инволюцию на множестве стабильных гомотопических типов: все пространства, двойственные по Спеньеру-Уайтхеду стабильно гомотопически эквивалентным между собой пространствам, стабильно гомотопически эквивалентны между собой. Это сводит следствие 4 к теореме 6.

Использование симплициальных разрешений делает доказательство теоремы 6 особенно наглядным.

Действительно, рассмотрим проекцию $L^{\prime} \rightarrow \Upsilon(\mathscr{L})$, индуцированную стандартной проекцией пространства $\Upsilon(\mathscr{L}) \times L \supset L^{\prime}$. Все слои этого отображения являются плоскостями $L_{I}$ для подходящих множеств $I$. Гомотопическая эквивалентность $L^{\prime} \sim \Upsilon(\mathscr{L})$ доказьвается индукцией по последовательному стягиванию этих слоев над стратами $\Upsilon(\mathscr{L})$, ср. с леммой 1 в $\S$ III.3.4 [84]; на каждом шаге гомотопическая эквивалентность следует из леммы Борсука.

Чтобы доказать (12), используем разновидность индукции из $\S 6$. Для любого $t>0$ обозначим через $\bar{L}(t)$ факторпространство $L /(L \cap\{x: f(x) \geqslant t\})$. Тогда для достаточно малых $t$ имеем $\bar{L}(t)=\{$ точка $\}$; для достаточно больших $t \bar{L}(t)$ гомотопически эквивалентно $\bar{L}$. Если отрезок $[a, b]$ не содержит критических значений, то $\bar{L}(a)$ гомотопически эквивалентно $\bar{L}(b)$, поэтому нам остается лиш доказать следующую лемму.

ЛЕмма 1. Если отрезок $[a, b]$ содержит лишь одно критическое значение функции $f$, а именно, критическое значение ее ограничения на плоскость $L_{I}$, то имеется гомотопическая әквивалентность

$$
\bar{L}(b) \sim \bar{L}(a) \vee \Sigma^{\operatorname{dim} L_{I}}(\Upsilon(I) / \partial \Upsilon(I)) .
$$

ДокАЗАтельство. Рассмотрим также пространства $\bar{L}^{\prime}(t)=L^{\prime} /\left(L^{\prime} \cap \pi^{-1}(\{x\right.$ : $f(x) \geqslant t\}))$. Проекция $\pi$ индуцирует гомотопические эквивалентности $\bar{L}^{\prime}(t) \sim \bar{L}(t)$, и нам достаточно доказать вариант леммы 1 , в котором $\bar{L}(b)$ и $\bar{L}(a)$ заменены соответственно на $\bar{L}^{\prime}(b)$ и $\bar{L}^{\prime}(a)$. В этом доказательстве используем топологическую операцию приклеивания топологических пространств по отображениям. Именно, если даны два топологических пространства $X, Y$, подпространство $A \subset X$ и непрерывное отображение $\varphi: A \rightarrow Y$, то пространство $Y \cup_{\varphi} X$ определяется как факторпространство несвязного объединения $X \sqcup Y$ по отношению $a \sim \varphi(a)$ для всех $a \in A$. В частности, если $Z=X \cup Y$, то $Z$ можно рассматривать как $X$, приклеенное к $Y$ 
по тождественному вложению $X \cap Y \rightarrow Y$. Важное свойство этой операции состоит в ее гомотопической инвариантности: любая гомотопическая эквивалентность $f: Y \rightarrow Y^{\prime}$ индуцирует гомотопическую эквивалентность

$$
Y \cup_{\varphi} X \sim Y^{\prime} \cup_{f \circ \varphi} X
$$

Для любой плоскости $L_{J}$ нашего набора определим $L_{J}^{\prime}$ как ее собственный прообраз, т.е. множество $\Upsilon(J) \times L_{J} \subset \Upsilon(\mathscr{L}) \times L$. Пусть $L ! a \subset L^{\prime}$ - это объединение собственных прообразов всех плоскостей $L_{J}$ таких, что $L_{J} \cap\{x: f(x)<a\} \neq \varnothing$. В частности, $L ! b=L_{I}^{\prime} \cup L ! a$.

Для любого подмножества $X \subset L^{\prime}$, обозначим через $X_{/ t}$ его приведение по модулю множества таких точек $x$, что $f \circ \pi(x) \geqslant t$. Тогда

$$
\bar{L}^{\prime}(b)=(L ! b)_{/ b}=\left(L_{I}^{\prime}\right)_{/ b} \cup(L ! a)_{/ b} \equiv\left(L_{I}^{\prime}\right)_{/ b} \cup_{\mathrm{id}}(L ! a)_{/ b},
$$

где id - это тождественное вложение $\left(L_{I}^{\prime}\right)_{/ b} \cap(L ! a)_{/ b} \rightarrow \cup(L ! a) / b$.

ЛЕмма 2. Имеется гомотопическая эквивалентность $(L ! a)_{/ b} \rightarrow(L ! a)_{/ a} \equiv$ $\bar{L}^{\prime}(a)$, индуиированная приведением по модулю слоя $\{x: f \circ \pi(x) \in[a, b]\}$.

Эта гомотопическая эквивалентность отображает все множество $\left(L_{I}^{\prime}\right) / b \cap(L ! a) / b$ в одну точку (полученную при факторизации из этого слоя). Следовательно, в силу (15) пространство (16) гомотопически эквивалентно букету $\bar{L}^{\prime}(a)$ и факторпространства $\left(L_{I}^{\prime}\right) / b /\left(\left(L_{I}^{\prime}\right) / b \cap(L ! a) / b\right)$.

Но последнее пространство $\left(L_{I}^{\prime}\right) / b /\left(\left(L_{I}^{\prime}\right) / b \cap(L ! a)_{/ b}\right)$ гомотопически эквивалентно $\left(\Upsilon(I) \times L_{I}\right) /\left(\left(\Upsilon(I) \times\left(L_{I}\right)\right) / b \cup\left(\partial \Upsilon(I) \times L_{I}\right)\right) \sim \Sigma^{\operatorname{dim} L_{I}}(\Upsilon(I) / \partial \Upsilon(I))($ cp. $[76]) ;$ лемма 1 и теорема 6 доказаны.

\section{§. Примеры: разрешения важных наборов плоскостей. Комплексы связных графов и гиперграфов}

Рассмотрим вновь диагональный набор плоскостей $A(N, 2)$ в $\mathbb{C}^{N}$ или $\mathbb{R}^{N}$, см. $\S 2$, и его наивное симплициальное разрешение $L^{\prime} \subset \Delta(\mathscr{L}) \times L$. Наименьшая плоскость $L_{I}$ этого набора - это прямая $\left\{x_{1}=\cdots=x_{N}\right\}$. Прообраз любой ее точки - это весь симплекс $\Delta \equiv \Delta(\mathscr{L}),\left(\begin{array}{c}N \\ 2\end{array}\right)$ вершин которого соответствуют всем возможньм гиперплоскостям $V_{i j} \equiv\left\{x_{i}=x_{j}\right\}$.

Нарисуем где-нибудь $N$ точек, помеченных номерами $1, \ldots, N$. Удобно изображать любую гиперплоскость $V_{i j}$ отрезком, соединяющим точки $i$ и $j$. Любая грань симплекса $\Delta$ определяет граф, состояший из отрезков, соответствуюших всем вершинам этого симплекса. Легко видеть, что подкомплекс маргинальных граней $\partial \Delta(\mathscr{L})$ симплекса $\Delta(\mathscr{L})$ состоит из всех граней, соответствуюших несвязньм графам. Поэтому гомологии диагонального набора связаны с группой гомологий комплекса связных графов, определяемого как факторкомплекс стандартной (ацикличной) триангулящии симплекса $\Delta$ по подкомплексу, натянутому на все грани, соответствуюшие несвязньпм графом.

ПРЕДЛОЖЕНИЕ 7 (см. [76]). Комплекс связных графов с $N$ вериинами ацикличен во всех размерностях кроме $N-2$. Его группа $(N-2)$-мерных гомологий изоморфна $\mathbb{Z}^{(N-1) !}$ и свободно порожсдена классами всех змеевидных (т.е. гомеоморфных отрезку) деревьев, один из концов которых фиксирован. 
Первое утверждение этого предложения является частным случаем теоремы Фолкмана о гомологиях геометрических решеток [32].

ЗАмечАниЕ. Число $(N-1)$ ! уже возникало в этой работе. Действительно, в силу предложения 2 группа $H^{N-1}(M(N, 2))(N-1)$ !-мерна. В формуле Горески-Макферсона этагруппа соответствует слагаемому $H_{N-1}(\Upsilon(I), \partial \Upsilon(I)) \equiv H_{N-1}(\Delta(I), \partial \Delta(I))$, где $I$ - все множество $\{1, \ldots, N\}$.

Красивое описание групшы когомологий этого же комплекса дано в [69].

Естественное обобщение этого комплекса дают комплексы $i$-связных графов, см. $[78],[9],[70],[71]$. Они также имеют важные приложения в дифференциальной и гомотопической топологии.

Аналогично, если мы рассмотрим набор $k$-совпадений $A(N, k)$ в $\mathbb{R}^{N}$ или в $\mathbb{C}^{N}$ (см. $\S 2)$, то наименьшей плоскостью $L_{I}$ вновь будет прямая $\left\{x_{1}=\cdots=x_{N}\right\}$. Соответствуюший симплекс $\Delta$ имеет $\left(\begin{array}{c}N \\ k\end{array}\right)$ вершин, его грани соответствуют $k$-гunepграфам с $N$ вершинами $1, \ldots, N$, а маргинальньй подкомплекс $\partial \Delta \subset \Delta$ - это комплекс несвязных гиперграфов. Групша гомологий этого комплекса (и дополнения $M(N, k)$ к этому набору) изучалась А. Бьёрнером и $\Phi$. Велкером [17], см. также [16], [19].

В частности, они доказали следующее.

Теорема 7 (см. [17]). Для любого $k \geqslant 2$ симплекс $c\left(\begin{array}{c}N \\ k\end{array}\right)$ вериинами, приведенный по модулю обгединения граней, соответствующих несвязным $k$-гиперграфам, гомотопически эквивалентен букету сфер, в частности, все его группь гомологий не имеют кручения. Более того, эти группь могут быть нетривиальны только в размерностях вида $N-(k-2) t-2,1 \leqslant t \leqslant N / k$. Ранги этих групп кратнь $\left(\begin{array}{c}N-1 \\ k-1\end{array}\right)$, а в старшей возможсной размерности $d=N-k$ ранг $H_{N-k}$ равен $\left(\begin{array}{c}N-1 \\ k-1\end{array}\right)$.

Общая формула для этих рангов также была найдена в [17] (см. там теорему 4.5), но она намного сложнее.

ЗАмечАнИЕ (см. [19]). Топология вешественного множества $M(N, k) \subset \mathbb{R}^{N}$ дает хорошие оценки в следуюшей олимпиадной задаче. Пусть у нас есть $N$ монет, некоторые из которых фальшивы, и чашечные весы. Естественно предположить, что все подлинные монеты весят одинаково, а веса всех фальшивых отличны друг от друга и от веса подлинных монет. Для данного $k \geqslant 2$ сколько взвешиваний достаточно сделать, чтобы убедиться, что хотя бы $k$ из наших монет - настоящие?

Действительно, каждое взвешивание разделяет пространство $\mathbb{R}^{N}$ всех возможных наборов весов на три выпуклых множества: два полупространства и разделяющую их гиперплоскость. Любая последовательность взвешиваний вместе с их результатами выделяет некоторую клетку (быть может, пустую) в $\mathbb{R}^{N}$. Правильная стратегия решения нашей задачи (такие стратегии называются деревьями принятия решения decision trees) должна разбить наше пространство $\mathbb{R}^{N}$ на такие клетки, каждая из которых принадлежит либо набору $A(N, k)$, либо его дополнению $M(N, k)$. Следовательно, число образующих полной групш гомологий любого из этих пространств дает нижнюю оценку числа клеток, а следовательно, и сложности решаюшего дерева.

Среди истоков теории наборов плоскостей есть еще один класс олимпиадных задач - задачи о разрезании пирога, см. [93]. 


\section{$\S 10$. Комбинаторная реализация классов когомологий дополнений к наборам плоскостей}

Формула Горески-Макферсона (8) имеет следующую явную реализацию (найденную по существу в [96], приводимую здесь интерпретацию см. в [55]). Допустим, что в $\mathbb{R}^{N}$ задана евклидова метрика.

Рассмотрим постоянное векторное поле $V$ (“силу") в $\mathbb{R}^{N}$. Для любого $r$-мерного симплекса порядкового подкомплекса $\Upsilon(I) / \partial \Upsilon(I)$ (т.е. для строго убывающей последовательности из $r+1$ плоскостей $\left.L_{I_{1}} \supset L_{I_{2}} \supset \cdots \supset L_{I_{r}} \supset L_{I}\right)$ и любой точки $x \in L_{I}$ рассмотрим последовательность из $r+1$ лучей в $\mathbb{R}^{N}$, вытекаюших из $x$, а именно, траектории точки $x$ в плоскостях $\mathbb{R}^{N}, L_{I_{1}}, \ldots, L_{I_{r}}$ под действием этой силы. (Мы можем рассматривать $V$ как градиентное поле линейной функции $\theta: \mathbb{R}^{N} \rightarrow \mathbb{R}$, тогда эти лучи будут траекториями градиентов ограничений $\theta$ на эти плоскости.)

ОПРЕДЕЛЕНИЕ. Постоянное векторное поле $V$ - типичное по отношению к набору плоскостей $L$, если для любого $I$ и любого симплекса в $\Upsilon(I) / \partial \Upsilon(I)$ эти лучи линейно независимы в $\mathbb{R}^{N}$.

Легко видеть, что такие векторные поля образуют открытое плотное подмножество в пространстве $\mathbb{R}^{N}$ всех постоянных полей. Пусть наше поле $V$ типично. Тогда для любого $I$ и симплекса как вьше вьпуклая оболочка наших $r+1$ лучей линейно гомеоморфна $(r+1)$-мерному октанту с вершиной в точке $x$. Такие октанты, соответствуюшие всем точкам $x \in L_{I}$, заметают $\left(r+1+\operatorname{dim} L_{I}\right)$-мерный клин в $\mathbb{R}^{N}$.

Если мы имеем $r$-мерный цикл $\alpha$ комплекса $\Delta(I) / \partial \Delta(I)$, то сумма (единообразно ориентированных) соответствуюших клиньев является относительным циклом в $\mathbb{R}^{N}$ $(\bmod L)$, и относительньй гомологический класс $\nabla \alpha \in H_{r+1+\operatorname{dim} L_{I}}\left(\mathbb{R}^{N}, L\right)$ последнего цикла зависит только от класса $\alpha$ в $H_{*}(\Delta(I), \partial \Delta(I))$ (с точностью до знака, зависящего от выбора ориентации плоскости $\left.L_{I}\right)$.

Наконец, мы берем класс в $H^{*}\left(\mathbb{R}^{N} \backslash L\right)$, двойственный по Пуанкаре-Лефшецу к $\nabla \alpha$ в многообразии $\mathbb{R}^{N} \backslash L$, т.е. определенный индексами пересечения с относительным циклом $\nabla \alpha$.

Эта реализация формулы (8) зависит от выбора направления $V$, но не очень сильно. Два элемента в $\bar{H}_{*}\left(\mathbb{R}^{N}, L\right)$, соответствуюших таким образом одному и тому же классу $\alpha \in H_{*}(\Delta(I), \partial \Delta(I))$ при помощи разных векторных полей, могут различаться только на элементы меньшей фильтрации: точнее, на суммы аналогичных классов, приходящих из слагаемых $H_{*}(\Delta(J), \partial \Delta(J))$, соответствующих плоскостям $L_{J}, c m p o$ го содержащим $L_{I}$.

Более того, если коразмерности всех плоскостей $L_{I}$ во всех бо́льших плоскостях $L_{J}$ не меньше двойки, то изоморфизм (8) каноничен (с точностью до выбора ориентаций всех плоскостей $\left.L_{I}\right)$ : действительно, в этом случае пространство типичных векторных полей $V$ линейно связно.

По аналогии с теорией узлов (ср. [63], [81]), такие реализации элементов групшы $H^{*}\left(\mathbb{R}^{N} \backslash L\right)$ можно назвать их комбинаторныцми представлениями.

Эта конструкция позволяет исследовать мультипликативную структуру в кольце $H^{*}\left(\mathbb{R}^{N} \backslash L\right)$, в частности полностью описать эту структуру в присоединенном градуированном кольце. 


\section{§11. Умножение в когомологиях}

Перепишем равенство (8) как равенство для ассоциированных градуированных групп:

$$
G r H^{*}\left(\mathbb{R}^{N} \backslash L\right) \cong \bigoplus H_{k-*-1-\operatorname{dim} L_{I}}(\Upsilon(I), \partial \Upsilon(I)) .
$$

Этот изоморфизм каноничен (с точностью до выбора ориентаций плоскостей $L_{I}$ ), a умножение в присоединенном градуированном кольие устроено следующим образом.

Рассмотрим две плоскости $L_{I}, L_{J} \subset L$ и два цикла $A, B$ факторкомплексов $\Upsilon(I) / \partial \Upsilon(I)$ и $\Upsilon(J) / \partial \Upsilon(J), \operatorname{dim} A=u, \operatorname{dim} B=v$, представленных цепями (= линейньми комбинациями симплексов) подкомплексов $\Upsilon(I), \Upsilon(J)$ с гранищами в $\partial \Upsilon(I)$ и $\partial \Upsilon(J)$. Тасовочное произведение $A \diamond B$ этих циклов определяется следуюшим образом (см. [91]).

Если $L_{I}$ и $L_{J}$ не трансверсальны (т.е. принадлежат некоторой собственной плоскости в $\mathbb{R}^{N}$ ) или не пересекаются, то $A \diamond B=0$. Допустим теперь, что $L_{I}$ и $L_{J}$ трансверсальны и $L_{K}=L_{I} \cap L_{J} \neq \varnothing$ (мы можем положить $K=I \cup J$ ). Пусть $a \subset A$ и $b \subset B$ - некоторые два симплекса с $u+1$ и $v+1$ вершинами соответственно, т.е. некоторые убывающие последовательности плоскостей пересечения набора $L$, оканчивающиеся элементами $\left\{L_{I}\right\}$ и $\left\{L_{J}\right\}$. Рассмотрим все $\left(\begin{array}{c}u+v+2 \\ u+1\end{array}\right)$ возможных тасовок этих последовательностей, т.е. всех (немонотонных) последовательностей из $u+v+2$ плоскостей, в которых встречаются все элементыпоследовательностей $a$ и $b$, сохраняя порядок, который они имели в этих последовательностях $a$ и $b$. Любой такой тасовке соответствует некоторая монотонная последовательность: любой элемент $\lambda$ тасовки, приходяший из последовательности $a$ (соответственно, $b$ ) заменяется на пересечение соответствуюшей плоскости с последней плоскостью, приходящей из последовательности $b$ (соответственно, $a$ ) и стояшей раньше, чем $\lambda$ в тасовке. Полученная монотонная последовательность по определению является $(u+v+1)$-мерным симплексом порядкового комплекса $\Upsilon(K)$. Тасовочное произведение наших симплексов $a$ и $b$ определяется как сумма всех таких симплексов, взятых со знаками, равными четностям соответствуюших тасовок (т.е. числам перестановок, сводяших их к последовательной записи последовательностей $a$ и $b$ ), и умноженных еще на один знак, которьй зависит только от мультииндексов $I, J$ и $K$ и определяется сравнением фиксированной коориентации плоскости $L_{K}$ в $\mathbb{R}^{N}$ с упорядоченной парой коориентаций плоскостей $L_{I}$ и $L_{J}$. Тасовочное произведение циклов $A$ и $B$ определяется по линейности. Оно является относительньм циклом, определяющим некоторьй элемент слагаемого в правой части (17), соответствуюшего плоскости $L_{K}$; этот элемент зависит только от классов гомологий шиклов $A$ и $B$ в слагаемых, соответствуюших $L_{I}$ и $L_{J}$.

Теорема 8 (см. [91], [92], [28], [29]). Изоморфизм (17) сопрягает тасовочное произведение в его правой части и умножение в его левой части, полученное из обичного когомологического умножения. Если все плоскости $L_{I}$ имеют коразмерности $\geqslant 2$ во всех бо́льших плоскостях $L_{I^{\prime}}$, то это жее верно для изоморфизма (8) и умножения в само́м кольце $H^{*}\left(\mathbb{R}^{m} \backslash L\right)$, а не только в его градуированном кольце.

Это следует из описанной в предыдушем параграфе явной реализации классов относительных гомологий. Действительно, умножение в когомологиях ориентированного многообразия $M$ можно реализовать следуюшим образом. Пусть даны два класса $\alpha, \beta \in H^{*}(M)$. Возьмем циклы Бореля-Мура $[\alpha],[\beta]$, двойственные к ним по Пуанкаре и пересекающиеся трансверсально. Возьмем их пересечение $[\alpha] \cap[\beta]$, снабженное 
естественной ориентацией, и рассмотрим класс когомологий, двойственный по Пуанкаре к этому пересечению.

Если даны плоскости $L_{I}, L_{J}$ и классы $\alpha \in H_{*}(\Upsilon(I), \partial \Upsilon(I)), \beta \in H_{*}(\Upsilon(J), \partial \Upsilon(J))$, то мы можем реализовать соответствуюшие элементы в левых частях (8) с помошњю разных постоянных векторных полей $V_{I}, V_{J}$ в $\mathbb{R}^{N}$, находяшихся в обшем положении друг к другу, если $L_{I}$ и $L_{J}$ пересекаются трансверсально; если же нет, то эти направления должны быть противоположны друг другу и трансверсальны гиперплоскости, разделяющей или содержащей эти плоскости.

Предложение 4 немедленно вытекает из теоремы 8.

УПРАЖнЕниЕ. Вывести теорему 2 (Орлика-Соломона) из этой теоремы.

\section{§ 12. Комплекс Сальветти для комплексифицированного набора вещественных гиперплоскостей}

Рассмотрим комплексификацию набора аффинных вешественных гиперплоскостей, т.е. набор комплексных гиперплоскостей $L_{j} \subset \mathbb{C}^{N}, j=1, \ldots, m$, выделенных теми же самыми линейньми уравнениями $f_{j}(x)=0, f_{j}\left(\mathbb{R}^{N}\right)=\mathbb{R}$. Его дополнение $\mathbb{C}^{N} \backslash L$ является $N$-мерным многообразием Штейна, в частности, гомотопически эквивалентно клеточному комплексу размерности $\leqslant N$. М. Сальветти [65], следуя некоторым идеям из [26], явно построил $N$-мерньй симплициальньй комплекс, вложенньй в пространство $\mathbb{C}^{N} \backslash S$ как его деформационньй ретракт. Здесь мы дадим простое описание этой конструкции в терминах двойственного комплекса.

Для каждой из наших плоскостей $L_{j}$ ее дополнение $\mathbb{C}^{N} \backslash L_{j}$ разделяется на четыре клетки $+_{j},-{ }_{j}, \uparrow_{j}$ и $\downarrow_{j}$, заданные, соответственно, условиями $\operatorname{Re} f_{j}>0, \operatorname{Re} f_{j}<0$, $\left\{\operatorname{Re} f_{j}=0, \operatorname{Im} f_{j}>0\right\}$ и $\left\{\operatorname{Re} f_{j}=0, \operatorname{Im} f_{j}<0\right\}$. Каждой из $4^{m}$ возможных последовательностей $m$ знаков,,$+- \uparrow$ и $\downarrow$ поставим в соответствие пересечение соответствующих клеток (например, $\left.\left(+_{1}\right) \cap\left(\uparrow_{2}\right) \cap\left(-_{3}\right) \cap \cdots \cap\left(\uparrow_{m}\right)\right)$. Это пересечение нескольких вешественных аффинных плоскостей и открытых полупространств в $\mathbb{C}^{N}$ гомеоморфно клетке. По определению оно лежит в $\mathbb{C}^{N} \backslash L$, и любая точка множества $\mathbb{C}^{N} \backslash L$ принадлежит ровно одной из этих клеток.

Лемма 3. Разбиение многообразия $\mathbb{C}^{N} \backslash L$ на клетки, соответствующие всевозмо жным последовательностям знаков,,$+- \uparrow u \downarrow$, дополненное одной 0-мерной клеткой, определяет клеточную структуру на одноточечной компактификации әтого многообразия.

Доказательство элементарно, ср. [33].

Комплекс Сальветти (как топологическое пространство) - это просто комплекс, двойственньй к этому клеточному разбиению. Как комбинаторньй объект он определяется некоторьм подразбиением этого двойственного комплекса.

Эта конструкция была использована в [36] для построения топологических инвариантов абстрактных ориентированных матроидов (см. 14 и [13]). См. также [18].

Описанное выше клеточное разбиение многообразия $\mathbb{C}^{N} \backslash L$ можно сильно упростить, если наш набор гиперплоскостей имеет только нормальные пересечения. В этом случае каждой плоскости $L_{I}$, выделенной несколькими уравнениями $f_{i}=0, i \in I$, $f_{i}\left(\mathbb{R}^{N}\right) \subset \mathbb{R}$, сопоставим мнимый конус в $\mathbb{C}^{N}$, выделенный условиями $\left\{\operatorname{Re} f_{i}=0\right.$, $\left.\operatorname{Im} f_{i}>0\right\}, i \in I$. Обозначим через $\nabla_{I}$ этот конус, из которого выброшены его пересечения с $L$ и со всеми меньшими конусами $\nabla_{J}, J \supsetneqq I$. 
ЛЕмма 4 (см. [88]). Если $L$ - комплексификация набора вещественных гиперплоскостей с нормальными пересечениями, то любое непустое множество $\nabla_{I}$ гомеоморфно клетке размерности $2 N-\# I$, и все эти мноэсества вместе образуют клеточное разбиение одноточечной компактификации многообразия $\mathbb{C}^{N} \backslash L$

Все коэффищиенты инцидентности соответствующего клеточного комплекса тривиальны, следовательно, группа гомологий Бореля-Мура $\bar{H}_{*}\left(\mathbb{C}^{N} \backslash L\right)$ свободная абелева, причем ранг группы $\bar{H}_{2 N-p}$ равен числу непустых плоскостей $L_{I}$ с \#I=p. Конечно, последнее утверждение вытекает также из формулы Горески-Макферсона, но конусы $\nabla_{I}$ дают особенно простую ее реализацию.

О комбинаторных и топологических свойствах наборов гиперплоскостей см. также [14]-[18], [34]-[39], [43], [57]-[60], [72], [73], [87], [88], [93]-[96].

\section{$\S$ 13. Гомологии дополнений к наборам плоскостей с подкрученными коэффициентами. Резонансы}

Ситуащия немного усложняется, если мы рассматриваем групшы гомологий с коэффициентами в локальных системах.

ОПРЕДЕЛЕНИЕ. Линейная локальная система на (локально односвязном) топологическом пространстве $X$ (например, на $X=\mathbb{C}^{N} \backslash L$ ) - это векторное расслоение $\pi: M \rightarrow X$ со слоем $\mathbb{C}^{1}$, снабженное плоской связностью, уважающей структуру $\mathbb{C}$-модуля в слоях.

Иными словами, для любой точки $x \in X$, любой достаточно малой окрестности $U$ точки $x$ в $X$ и любой точки $a \in \pi^{-1}(x)$ мы имеем выделенное сечение нашего расслоения над всем $U$, равное $a$ в $x$; это сечение будет тем же самым, если мыначнем с любой другой точки $x^{\prime} \in U$ и точки пересечения $a^{\prime}$ старого сечения со слоем $\pi^{-1}\left(x^{\prime}\right)$. Для любых двух точек $a_{1}, a_{2}$ слоя $\pi^{-1}(x)$ выделенное сечение, равное $a_{1}+a_{2}$ в точке $x$, состоит из послойных сумм образов сечений, равных $a_{1}$ и $a_{2}$ в точке $x$, а для любого $\lambda \in \mathbb{C}$ сечение, равное $\lambda a$ в точке $x$, состоит из умноженных на $\lambda$ образов сечения, равного $a$ в $x$.

$i$-мерньй сингулярный симплекс локальной системы $\Theta$ - это непрерьвное отображение стандартного симплекса $\Delta^{i}$ в тотальное пространство $M$ расслоения, уважаюшее плоскую связность: если точка $\xi$ симплекса переходит в некоторую точку $a \in \pi^{-1}(x), x \in X$, то некоторая малая окрестность $\xi$ переходит в образ соответствуюшего сечения над малой окрестностью точки $x$. Группа $i$-мерных сингулярных цепей с коэффициентами в локальной системе $\Theta$ определяется как факторгруппа абелевой группы, порожденной всеми такими локально горизонтальными отображениями $\Delta^{i} \rightarrow M$, по следуюшим соотношениям:

а) если имеются два сингулярных симплекса $\varphi_{1}, \varphi_{2}: \Delta^{i} \rightarrow M$ с одной и той же проекцией (т.е. $\pi \circ \varphi_{1} \equiv \pi \circ \varphi_{2}$ ), то их сумма $\varphi_{1}+\varphi_{2}$ равна третьему сингулярному симплексу $\varphi_{3}$ такому, что $\varphi_{3}(\xi)=\varphi_{1}(\xi)+\varphi_{2}(\xi)$ для любого $\xi \in \Delta^{i}$;

б) для любого $\lambda \in \mathbb{C}$ сингулярньй симплекс $\varphi$, взятый с коэффищиентом $\lambda$, равен сингулярному симплексу, отображающему любую точку $\xi$ в $\lambda \varphi(\xi)$.

Граница такого симплекса определяется очевидным образом и является суммой сингулярных симплексов (меншшей размерности) этой же локальной системы. Мы можем рассматривать комплекс конечных цепей (т.е. конечных линейных комбинаций сингулярных симплексов) или локально конечных цепей (т.е., вообще говоря, бесконечных линейных комбинаций, проекции которых в $X$ образуют там локально конеч- 
ные цепи). Соответствуюшие группы гомологий будут обозначаться, соответственно, через $H_{i}(X, \Theta)$ и $H_{i}^{\mathrm{lf}}(X, \Theta)$; они называются группами гомологий $X$ с коэффиииентами в локальной системе $\Theta$ или просто гомологиями локальной системь $\Theta$.

Эти групшы связаны каноническим гомоморфизмом

$$
H_{i}(X, \Theta) \rightarrow H_{i}^{\mathrm{lf}}(X, \Theta)
$$

В частности, если наше расслоение $M$ - это прямое произведение $X \times \mathbb{C}^{1}$ с очевидным плоским сечением, то мы получаем, соответственно, обычную групу гомологий $H_{i}(X, \mathbb{C})$ и группу гомологий Бореля-Мура $\bar{H}_{i}(X, \mathbb{C})$. Группы когомологий $H^{i}(X, \Theta), H_{\mathrm{lf}}^{i}(X, \Theta)$ определяются стандартньм образом как группы гомологий сопряженных комплексов.

Любая линейная локальная система очевидным образом определяет гомоморфизм монодромии фундаментальной группы $\pi_{1}(X)$ (или, эквивалентно, группы $\left.H_{1}(X)\right)$ в группу $\operatorname{Aut}\left(\mathbb{C}^{1}\right) \equiv \mathbb{C}^{*}:$ продолжая наши сечения над замкнутой петлей в $X$, мы действуем в слоях умножением на коэффициент монодромии, соответствуюший этой петле.

Любой локальной системе $\Theta$ соответствует ее двойственная система $\Theta^{*}$, слои которой отождествлены с пространствами $\mathbb{C}$-линейных функций на слоях исходной системы, и это отождествление согласовано с плоскими сечениями в обеих. Коэффициенты монодромии, заданные одним и тем же элементом группы $\pi_{1}(X)$ во взаимно двойственных системах, обратны друг другу (т.е. их произведение равно 1). Если пространство $X$ является $d$-мерным ориентированным многообразием, то его группы гомологий и когомологий с коэффициентами в двойственных локальных системах связаны изоморфизмами Пуанкаре

$$
H_{i}^{\mathrm{lf}}(X, \Theta) \simeq H^{d-i}\left(X, \Theta^{*}\right), \quad H_{\mathrm{lf}}^{i}(X, \Theta) \simeq H_{d-i}\left(X, \Theta^{*}\right) .
$$

(Более того, замечательная локальная система Or, называемая ориентирующим пучком, позволяет задать изоморфизмы Пуанкаре на неориентированных многообразиях: это верно, даже если $\Theta$ и $\Theta^{*}$ - постоянные локальные системы. А именно, в любом из двух уравнений (19) правую часть надо заменить на аналогичное выражение, в котором коэффициентная локальная система - не $\Theta^{*}$, а $\Theta^{*} \otimes$ Or: ее коэффициенты монодромии совпадают с такими же коэффициентами для $\Theta^{*}$ с точностью до знака, равного + или - в зависимости от того, сохраняет ли соответствующая петля ориентацию многообразия $X$ или нет.)

Локальные системы и их группы гомологий являются адекватньм инструментом при анализе ветвяшихся дифференциальных форм и их интегралов, см. [25]. Пусть у нас есть замкнутая аналитическая $i$-форма на $X$ такая, что аналитическое продолжение вдоль любого замкнутого пути $c$ в $X$ умножает ее на комплексное число, причем это число $\tau(c)$ зависит только от класса нашего пути $c$ в группе $H_{1}(X)$. (Важньй класс таких форм будет рассмотрен в $\S 15$.) Тогда контуры интегрирования этих дифференциальных форм являются корректно определенными элементами группы $i$-мерных гомологий $X$ с коэффициентами в некоторой локальной системе, коэффициент монодромии которой на любом пути $c$ равен $1 / \tau(c)$.

Теперь пусть имеется набор гиперплоскостей $\mathscr{L}=\left\{L_{1}, \ldots, L_{m}\right\}$ в $\mathbb{C}^{N}, m \geqslant N$, $X=\mathbb{C}^{N} \backslash L$ и линейная локальная система $\Theta$ на $X$; обозначим через $\tau_{1}, \ldots, \tau_{m}$ коэффициенты монодромии этой системы, соответствующие маленьким окружностям, обходящим вокруг этих гиперплоскостей в положительном направлении. 
Теорема 9 (см. [88], [7], [86]). Пусть $\mathscr{L}$ - набор гиперплоскостей общего положения в $\mathbb{C}^{N}$. Тогда справедливы следующие утверждения.

А. Если хотя би один из коэффициентов $\tau_{i}$ не равен 1, то группь $H_{i}(X, \Theta)$, $H_{i}^{\mathrm{lf}}(X, \Theta)$ нетривиальнь только при $i=N$, и их размерности равньи $\left(\begin{array}{c}m-1 \\ N\end{array}\right)$.

Б. Отображсение (18) между әтими группами является изоморфизмом тогда и только тогда, когда все числа $\tau_{i}(i=1, \ldots, m)$ и их произведение $\tau_{0} \equiv \tau_{1} \cdots \tau_{m}$ не равньи 1 .

В. Если все эти числа отличны от 1 и набор $\mathscr{L}$ является комплексификацией вещественного, то группа $H_{N}^{\mathrm{lf}}\left(\mathbb{C}^{N} \backslash L, \Theta\right)$ свободно порожсдена классами всех ограниченных компонент мноэсества $\mathbb{R}^{N} \backslash L$.

В последнем случае комплексифицированного типичного вещественного набора гиперплоскостей эти факты (размерности обеих групп, биективность отображения (18) и утверждение пункта В) были вначале доказаны К. Аомото [2] при гораздо более сильных ограничениях на коэффициенты $\tau_{j}$.

Для нетипичных наборов плоскостей множество исключительных значений вектоpa $\boldsymbol{\tau} \equiv\left(\tau_{1}, \ldots, \tau_{m}\right)$, для которых отображение $(18)$ не биективно, устроено более сложно, см., например, теорему 10 ниже. Начиная с [88] такие значения называются резонансами нашей локальной системы.

Пример. Пусть $N=1$, а $\mathscr{L}$ - набор из $m$ различных точек $L_{1}, \ldots, L_{m}$. Если хотя бы один из соответствуюших коэффициентов $\tau_{i}$ отличен от 1 , то группа $H_{1}^{\mathrm{lf}}\left(\mathbb{C}^{1} \backslash L, \Theta\right)$ порождена классами любых $m-1$ из $m$ параллельных лучей $\nabla_{j}$, соединяющих эти точки с бесконечностью, см. рис. 5. Действительно, все эти $m$ лучей и дополнение к их объединению являются клетками, покрывающими все множество $\mathbb{C}^{1} \backslash L$. Мы можем вычислять группу $H_{*}^{\mathrm{lf}}\left(\mathbb{C}^{1} \backslash L, \Theta\right)$ с помощью этой клеточной структуры. Легко видеть, что при некотором естественном выборе образуюших этого клеточного комплекса (т.е. пар вида \{клетка, допустимое сечение локальной системы над ней $\}$ ) коэфффициенты инцидентности единственной 2-мерной клетки со всеми одномерными равны $\tau_{1}-1, \ldots, \tau_{m}-1$. Это дает групу $H_{*}^{\text {lf }}\left(\mathbb{C}^{1} \backslash L, \Theta\right)$; структура "абсолютной" группы $H_{*}\left(\mathbb{C}^{1} \backslash L, \Theta\right)$ получается при помощи двойственности Пуанкаре из аналогичного утверждения о группе $H_{*}\left(\mathbb{C}^{1} \backslash L, \Theta^{*}\right)$.

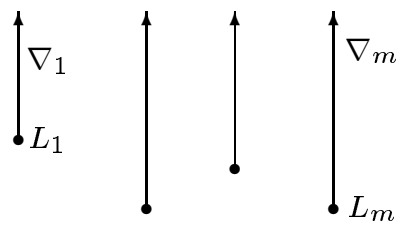

Рис. 5. Разбиение дополнения к набору точек в $\mathbb{C}^{1}$

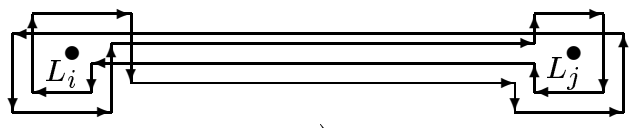

a)

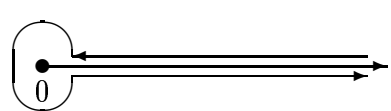

б)

Рис. 6. Цикл “двойная петля”

Если $\tau_{j}=1$, то маленькая окружность вокруг точки $L_{j}$ является нетривиальньм элементом групшы $H_{1}\left(\mathbb{C}^{1} \backslash L\right)$ (поскольку ее индекс пересечения с лучом $\nabla_{j}$ не равен 
нулю). С другой стороны, она, очевидно, гомологична нулю посредством локально конечной 2-цепи, ограниченной ею, следовательно, ядро отображения (18) нетривиально. Если $\tau_{1} \cdots \tau_{m}=1$, то это же верно для большой окружности, охватываюшей все точки $L_{j}$.

Если все числа $\tau_{1}, \ldots, \tau_{m}$ и их произведение $\tau_{0} \equiv \tau_{1} \cdots \tau_{m}$ отличны от 1 , то оператор, обратньй к (18), задается двойнымми петлями, т.е. циклами, изображенньми на рис. 6а). Такой цикл в $\mathbb{C}^{1} \backslash L$ обходит дважды (и в противоположных направлениях) вокруг каждой из наших двух особых точек $L_{i}, L_{j}$, поэтому он поднимается до цикла нашей локальной системы $\Theta$ и определяет элемент группы $H_{1}\left(\mathbb{C}^{1} \backslash L\right)$. Поскольку $\left(1-\tau_{i}\right)\left(1-\tau_{j}\right) \neq 0$, то образ этого цикла в $H_{1}^{\mathrm{lf}}\left(\mathbb{C}^{1} \backslash L\right)$ равен классу интервала $\left(L_{i}, L_{j}\right)$, взятому с ненулевым коэффициентом.

Теперь докажем теорему 9 в случае произвольного $N$.

А. Рассмотрим вновь универсальный $m$-набор гиперплоскостей, т.е. координатньй крест $\dagger^{m} \subset \mathbb{C}^{m}$, и локальную систему $\widetilde{\Theta}$ на $\mathbb{C}^{m} \backslash \dagger^{m}$ с теми же коэффициентами монодромии $\tau_{j}$. Если $\tau_{j} \neq 1$ для хотя бы одного $j$, то группа $H_{*}\left(\mathbb{C}^{m} \backslash \dagger^{m}, \widetilde{\Theta}\right)$ тривиальна во всех размерностях в силу теоремы Кюннета в $\left(\mathbb{C}^{m} \backslash t^{m}\right)=\left(\mathbb{C}^{*}\right)^{m}$. Наш типичньй набор $L \subset \mathbb{C}^{N}$ можно реализовать как прообраз $\dagger^{m}$ при некотором типичном аффинном вложении $\mathbb{C}^{N} \rightarrow \mathbb{C}^{m} ;$ наша локальная система $\Theta$ совпадает с системой, индуцированной из $\widetilde{\Theta}$ при этом вложении. В силу теоремы Лефшеца (см. [40]), наше вложение $\mathbb{C}^{N} \backslash L \rightarrow \mathbb{C}^{m} \backslash \dagger^{m} N$-связно, в частности, индуцирует изоморфизм груп гомологий во всех размерностях, меньших $N$. Следовательно, група $H_{i}\left(\mathbb{C}^{N} \backslash L, \Theta\right)$ тривиальна при $i<N$. В силу изоморфизма Пуанкаре (19), это же верно для всех групп $H_{i}^{\mathrm{lf}}\left(\mathbb{C}^{N} \backslash L, \Theta\right)$ с $i>N$. Размерность этих груп в единственной оставшейся размерности $N$ получается из изучения эйлеровой характеристики (которая не зависит от выбора системы $\Theta$ ).

Б. Если хотя бы одно из чисел $\tau_{j}$ равно 1 , то, так же как в одномерном примере, маленькая окружность, обходщая соответствующую плоскость $L_{j}$, задает нетривиальньй элемент ядра отображения (18). Если все числа $\tau_{j}$ и их произведение $\tau_{0}$ отличны от 1 , то биективность (18) вытекает из гораздо более общего утверждения.

ПРЕДЛОЖЕНИЕ 8. Пусть $W$ - компактное $N$-мерное комплексное алгебраическое многообразие и $\widetilde{L}$ - конечный набор гладких гиперповерхностей в нем, имеющих только нормальные пересечения. Пусть $\theta$ - линейная локальная система на $W \backslash \widetilde{L}$ такая, что все коэффичиенты монодромии, соответствуюшие маленьким окружностям, обходящим вокруг компонент $\widetilde{L}$, отличны от 1 . Тогда каноническое отображение $H_{*}(W \backslash \widetilde{L}, \theta) \rightarrow H_{*}^{\mathrm{lf}}(W \backslash \widetilde{L}, \theta)$ является изоморфизмом во всех размерностях.

В частности, если $W \backslash \widetilde{L}$ - многообразие Штейна, то обе групшы могут быть нетривиальны только в размерности $N$. Это предложение легко вытекает из спектральной последовательности Лерә для когомологий пучков, см., например, [7], [86] .

Наше утверждение о биективности (18) вытекает из этого предложения, если взять $W=\mathbb{C P}^{N}$ и $\widetilde{L}=$ объединение $L$ и несобственной плоскости.

B. Если наш набор гиперплоскостей является комплексификацией вешественного, то обращение отображения (18) визуализируется “многомерными двойными петлями", обобщающими рис. 6а). Их конструкция была анонсирована в [88] и описана в [86]. А именно, пусть $\Delta \subset \mathbb{R}^{N}$ - ограниченная связная компонента множества $\mathbb{R}^{N} \backslash L$. Соответствующая “двойная петля" - это $N$-мерное многообразие $\kappa(\Delta)$ вместе с некоторой его иммерсией $K: \kappa(\Delta) \rightarrow \mathbb{C}^{N} \backslash L$ такие, что 
а) эта иммерсия поднимается до отображения в пространство $M$ нашей локальной системы $\Theta$, локально плоского относительно ее связности; в частности, она определяет (с точностью до скалярного множителя, зависяшего от выбора этого поднятия) элемент группы $H_{N}\left(\mathbb{C}^{N} \backslash L, \Theta\right)$;

б) отображение (18) переводит этот элемент в гомологический класс компоненты $\Delta$, взятьй с некоторьм коэффициентом, который отличен от нуля тогда и только тогда, когда все коэффициенты монодромии $\tau_{j}$, соответствуюшие всем стенкам многогранника $\Delta$, отличны от 1 .

Этот цикл строится с помошью вложения пары $\left(\mathbb{C}^{N}, L\right)$ в пространство $\left(\mathbb{C}^{p}, \dagger^{p}\right)$ универсального $p$-набора гиперплоскостей, где $p$ - число стенок $\Delta$. Вначале мы строим иммерсию $\mathbb{R}^{1} \rightarrow \mathbb{C}^{1} \backslash\{0\}$, изображенную на рис. 6б): она совпадает с тождественным отображением на интервале $[+\varepsilon,+\infty)$, с отображением $\{x \rightarrow-x\}$ на интервале $(-\infty,-\varepsilon]$ и отображает отрезок $[-\varepsilon,+\varepsilon]$ в маленькую окружность, обходящую начало координат. Прямое произведение $p$ экземпляров таких иммерсий определяет иммерсию $\Xi_{p}: \mathbb{R}^{p} \rightarrow \mathbb{C}^{p} \backslash \dagger^{p}$, накрьвающую положительньй октант в $\mathbb{R}^{p} \subset \mathbb{C}^{p}$ с кратностью $2^{p}$.

Пусть $\psi_{i}, i=1, \ldots, p,-$ линейные функции $\mathbb{C}^{N} \rightarrow \mathbb{C}$, выделяющие гиперплоскости $L_{j}$, ограничивающие компоненту $\Delta$; их можно нормировать так, чтобы все они принимали положительные значения в $\Delta$. Эти функции $\left(\psi_{1}, \ldots, \psi_{p}\right)$ определяют вложение $\Psi: \mathbb{C}^{N} \rightarrow \mathbb{C}^{p}$. Искомый цикл $\kappa(\Delta)$ индуцируется этим отображением из универсальной иммерсии $\Xi_{p}$. А именно, $\kappa(\Delta)$ определяется как подмножество прямого произведения $\mathbb{R}^{p} \times\left(\mathbb{C}^{N} \backslash L\right)$, состоящее из таких пар $(t, x)$, что $\Xi_{p}(t)=\Psi(x)$. Иммерсия $K-$ это ограничение очевидной проекции $\mathbb{R}^{p} \times\left(\mathbb{C}^{N} \backslash L\right) \rightarrow \mathbb{C}^{N} \backslash L$.

ЗАмечАниЕ. Аналогичные “двойные петли” обрашают отображение (18) также и в наиболее общей ситуации, описанной в предложении 8 , но их конструкция гораздо более сложна, см. $§ .10$ в [86]. (Прямое построение таких циклов в некоторых интересных частных случаях было дано в [61].) В математическом анализе это обрашение называется регуляризацией несобственных интегралов, см. также 115 ниже.

Рассмотрим линейную функцию $f: \mathbb{R}^{N} \rightarrow \mathbb{R}$ общего положения, значения которой на всех нульмерных плоскостях $L_{J}$ различны, и поставим в соответствие каждой ограниченной компоненте $\Delta$ ее вершину $L(\Delta)$, в которой достигается верхняя грань функции $\left.f\right|_{\Delta}$. Пусть $\nabla(\Delta)-N$-мерный мнимый конус $\nabla_{J}$ в $\mathbb{C}^{N} \backslash L$ с вершиной в $L(\Delta)$, см. последний абзац $\S 12$. Легко показать, что число ограниченных связных компонент $\Delta$ равно $\operatorname{dim} H_{*}\left(\mathbb{C}^{N} \backslash L, \Theta\right)$ (доказательство более общего утверждения см. после теоремы 10 ниже). Утверждение В теоремы 9 является следствием следующего предложения.

ПРЕДЛОЖЕНИЕ 9 (см. [88]). Если все коэффициенты монодромии $\tau_{j}, j=$ $1, \ldots, m$, отличны от 1, то

а) группа $H_{N}\left(\mathbb{C}^{N} \backslash L, \Theta\right)$ свободно порождена классами “двойных петель" $\kappa(\Delta)$, соответствующих всем ограниченным компонентам $\Delta$;

б) группа $H_{N}^{\mathrm{lf}}\left(\mathbb{C}^{N} \backslash L, \Theta\right)$ свободно порожсдена классами мнимых конусов $\nabla(\Delta)$, соответствующих этим компонентам.

Мы докажем одновременно утверждение а) для нашей локальной системы $\Theta$ и утверждение б) для двойственной системы $\Theta^{*}$. Именно, поднимем конусы $\nabla(\Delta)$ в тотальное пространство $M^{*}$ последней системы. Тогда индексы пересечения 
$\left\langle\kappa\left(\Delta_{1}\right), \nabla\left(\Delta_{2}\right)\right\rangle$ корректно определены для всех пар компонент $\Delta_{1}, \Delta_{2}$. Легко вычисляется, что матрица, состоящая из всех таких индексов пересечения, треугольна с ненулевыми значениями на диагонали; в частности, она невырождена.

Большинство утверждений теоремы 9 и предложения 9 продолжаются на более общие ситуации. Однако в этих случаях множество резонансных значений $T=$ $\left(\tau_{1}, \ldots, \tau_{m}\right)$ становится более сложным.

А именно, рассмотрим множество $\widehat{L} \subset \mathbb{C P}^{N}$, состояшее из носителя $L \subset \mathbb{C}^{N}$ нашего набора и из несобственной плоскости. Если это множество не является дивизором с нормальными пересечениями, построим его разрешение $(W, \widetilde{L}) \rightarrow\left(\mathbb{C P}^{N}, \widehat{L}\right)$ в смыс-

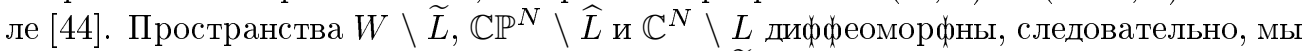
можем поднять локальную систему $\Theta$ на $W \backslash \widetilde{L}$. Для любой неприводимой компоненты $\widetilde{L}_{j}$ алгебраического множества $\widetilde{L}$ коэффициент монодромии, соответствующий маленькой охватьвающей ее окружности, является мономом от исходных коэффициентов $\tau_{1}, \ldots, \tau_{m}$. Наш набор $T=\left(\tau_{1}, \ldots, \tau_{m}\right)$ называется резонансныл, если значение какого-нибудь из этих мономов равно 1 при любом выборе разрешения множества $\widehat{L}$. Тривиальные мономы $\tau_{1}, \ldots, \tau_{m}$ и $\tau_{0}^{-1} \equiv\left(\tau_{1} \cdots \tau_{m}\right)^{-1}$ всегда возникают среди этих мономов: они соответствуют собственным прообразам исходных плоскостей $L_{j}$ и несобственной плоскости. Из предложения 8 немедленно следует, что если множество $T$ нерезонансно, то отображение (18) является изоморфизмом.

Tеорема 10 (см. [88]). Допустим, что наш набор зиперплоскостей $L \subset \mathbb{C}^{N}$ является комплексификацией вещественного, имеет только нормальные пересечения в $\mathbb{C}^{N}$ и все числа $\tau_{1}, \ldots, \tau_{m}$ отличны от 1 . Тогда справедливы следующие утверэдения.

А. Обе группьи $H_{i}\left(\mathbb{C}^{N} \backslash L, \Theta\right), H_{i}^{\mathrm{lf}}\left(\mathbb{C}^{N} \backslash L, \Theta\right)$ нетривиальньи только при $i=N$, и их размерности равнь числу ограниченных компонент множества $\mathbb{R}^{N} \backslash L$.

Б. Группа $H_{i}\left(\mathbb{C}^{N} \backslash L, \Theta\right)$ свободно порожсдена классами двойных петель, соответствующих всем этим компонентам, а группа $H_{i}^{\mathrm{lf}}\left(\mathbb{C}^{N} \backslash L, \Theta\right)$ свободно порождена классами соответствуюших мнимых конусов $\nabla(\Delta)$.

В. Если, более того, набор чисел $T=\left(\tau_{1}, \ldots, \tau_{m}\right)$ нерезонансньй, то группа $H_{i}^{\mathrm{lf}}\left(\mathbb{C}^{N} \backslash L, \Theta\right)$ свободно порождена также классами этих ограниченных компонент.

Доказательство утверждения А вытекает из клеточного разбиения, рассмотренного в конще $\S 12$. Действительно, соответствующий клеточньй комплекс, вычисляюший групу $H_{i}^{\mathrm{lf}}\left(\mathbb{C}^{N} \backslash L, \Theta\right)$, после некоторой нормировки его канонических образуюших оказьвается изоморфньм порядковому комплексу нашего набора плоскостей $\mathscr{L}$ или, эквивалентно, его вешественной части $\mathscr{L} \cap \mathbb{R}^{N} \equiv\left\{L_{j} \cap \mathbb{R}^{N}\right\}$. В силу формулы (11), последний комплекс гомотопически эквивалентен носителю $L \cap \mathbb{R}^{N}$, которьй очевидным образом гомотопически эквивалентен букету сфер, соответствуюших всем этим ограниченным компонентам. Доказательство утверждений Б и В - то же самое, что в случае типичных наборов.

ЗАмечАниЕ. В [25] был развит аналог теории де Рама, вычисляющий гомологии комплексных многообразий (например, пространств $\mathbb{C}^{N} \backslash L$ ) с коэффициентами в локальных системах, см. также [30], [2] и $\S 8$ в [90]. 


\section{§ 14. Матроиды и пространства конфигураций}

Простейший пример конфигурационного пространства - это пространство $M(N, 2)$ наборов из $N$ различных точек в $\mathbb{C}^{1}$, см. $\S 2$. В более сложных примерах мы можем рассматривать наборы алгебраических подмножеств в многообразиях; конфигурационное пространство - это семейство всех наборов таких, что пара (многообразие, объединение этих подмножеств) имеет фиксированный топологический тип. Такие конфигурационные пространства часто возникают в интегральной геометрии и теории спешиальных функций, см. [62], [34], [87], [86]. Важный класс таких функщий, называемых общими гипергеометрическими функииями, возникает, если все наши множества являются гиперплоскостями в $\mathbb{C}^{N}$ или в $\mathbb{C P}^{N}$, см. [34], [73]. В этом случае топология конфигурационных пространств имеет особенно глубокие связи с алгебраической геометрией. Удобно формулировать эти связи в терминах теории матроидов.

Понятие матроида является формализацией размерностных свойств центрального набора гиперплоскостей, см. [51] и [37].

ОПРЕДЕЛЕНИЕ. Матроид - это конечное множество $U$, снабженное натуральнозначной функцией $r$ на множестве $2^{U} \backslash\{\varnothing\}$ всех непустых подмножеств $U$ такой, что

1) для любого такого подмножества $I \in U$ имеем $1 \leqslant r(U) \leqslant$ (мошность множества $I)$;

2) если $I \subset J$, то $r(I) \leqslant r(J)$;

3) для любых $I, J$ имеем $r(I \cap J)+r(I \cup J) \leqslant r(I)+r(J)$.

Любой центральньй набор гиперплоскостей $\mathscr{L}=\left\{L_{1}, \ldots, L_{m}\right\}$ определяет матроид, элементы которого соответствуют плоскостям $L_{i}$, и для любого набора $I$ этих элементов $r(I)$ - это коразмерность пересечения $L_{I}$ всех плоскостей из этого набора.

В этом случае набор гиперплоскостей $\mathscr{L}$ назьвается реализацией соответствуюшего матроида.

Существуют матроиды, имеюшие комплексные реализации (т.е. реализации наборами комплексных гиперплоскостей в $\mathbb{C}^{N}$ ), но не имеющие вещественных, и матроиды, имеющие реализации над конечными полями, но не имеющие комплексных, и т. д. (С другой стороны, любая реализация матроида над некоторым полем определяет также его реализацию над любым расширением этого поля: в частности, комплексификация вещественной реализации доставляет комплексную реализацию.)

Специфические свойства наборов аффинных вешественных гиперплоскостей (грубо говоря, то, что пара 0 -мерных плоскостей $L_{I}, L_{I^{\prime}}$ может лежать либо по одну сторону от любой не содержащей их гиперплоскости, либо по разные) формализованы в понятии ориентированного матроида, см. [13].

Следующий важный вопрос таков: пусть дан матроид, как вьглядит множество всех его реализаций над данным полем $F$ ?

Изучение реализаций центральными наборами гиперплоскостей в $\mathbb{C}^{3}$ (или, эквивалентно, произвольными наборами прямых в $\mathbb{C P}^{2}$ ) тесно связано с теорией целочисленных алгебраических множеств (т.е. комплексных алгебраических множеств, заданных уравнениями с целочисленньми коэффициентами), см. [56], [57].

Согласно [56], [57], любому целочисленному алгебраическому подмножеству в некотором пространстве $\mathbb{C}^{n}$ соответствует такой матроид, что пространство его реализаций наборами плоскостей в $\mathbb{C}^{3}$ гомотопически эквивалентно этому подмножеству.

Эта связь позволяет построить пространства реализаций с весьма экзотическими свойствами: эти свойства отражают аналогичные свойства соответствующих алгебраических множеств. 
Например, уравнение $x^{2}=-1$ связано с матроидом, имеюшим комплексные реализации, но не имеюшим вешественных. Уравнение $x^{2}+y^{2}=0$ связано с таким матроидом, что вещественная размерность пространства его вешественных реализаций меньше комплексной размерности пространства его комплексных реализаций (в частности, последнее пространство негладко, см. [87]).

Первьй из этих примеров (соответствующий уравнению $x^{2}=-1$ ) строится следующим образом.

Первые четыре элемента $L_{1}, \ldots, L_{4}$ находятся в общем положении (т.е. $r(\{I\})$ равно 1 для любого 1-элементного множества $I \subset\{1,2,3,4\}, 2$ для любого 2-элементного множества и 3 для 3- и 4-элементных множеств). В терминах возможных реализаций прямыми в $\mathbb{C P}^{2}$ или в $\mathbb{R P}^{2}$ это означает, что эти прямые пересекаются обшим образом в обычном смысле: никакие три из них не пересекаются в одной точке. Если имеется реализация нашего матроида, включаюшего эти четыре элемента, то они фиксируют систему координат в $\mathbb{P}^{2}$ : мы можем взять $L_{1}$ в качестве прямой "на бесконечности", $L_{2}$ в качестве прямой $\{x=0\}, L_{3}$ в качестве прямой $\{y=0\}$ и выбрать масштаб координат так, чтобы точки $L_{2} \cap L_{4}$ и $L_{3} \cap L_{4}$ имели, соответственно, координаты $(0,1)$ и $(1,0)$.

Далее, добавим элемент $L_{5}$ с единственным условием необщего положения $r\left(L_{3}, L_{4}, L_{5}\right)=2$, т.е. соответствующие три прямые любой реализации в $\mathbb{P}^{2}$ должны пересекаться в одной точке. Точка пересечения этой прямой $L_{5}$ с $L_{2}$ будет иметь координаты $(0, \alpha)$, где $\alpha$ - какое-то число. Следующая прямая $L_{6}$ должна проходить через эту точку пересечения и быть параллельной к $L_{4}$ (в терминах матроида эти условия задаются так: $r\left(L_{2}, L_{5}, L_{6}\right)=2$ и $\left.r\left(L_{1}, L_{4}, L_{6}\right)=2\right)$. Координаты точки $L_{6} \cap L_{3}$ будут равны $(\alpha, 0)$. Следуюшая прямая $L_{7}$ проходит через эту точку и параллельна к $L_{5}$, т.е. мы имеем условия $r\left(L_{3}, L_{6}, L_{7}\right)=2$ и $r\left(L_{1}, L_{5}, L_{7}\right)=2$. Ее точка пересечения с прямой $L_{2}$ имеет координаты $\left(0, \alpha^{2}\right)$. Следуюшая прямая $L_{8}$ проходит через последнюю точку и параллельна к $L_{6}$ и $L_{4}$, т.е. имеем условия $r\left(L_{2}, L_{7}, L_{8}\right)=2$ и $r\left(L_{1}, L_{4}, L_{6}, L_{8}\right)=2$. Координаты точки ее пересечения с прямой $L_{3}$ равны $\left(\alpha^{2}, 0\right)$. Следуюшая прямая $L_{9}$ должна проходить через точки $(1,0)$ и $\left(0, \alpha^{2}\right)$, т.е. $r\left(L_{3}, L_{4}, L_{5}, L_{9}\right)=2$ и $r\left(L_{2}, L_{7}, L_{8}, L_{9}\right)=2$. Прямая $L_{10}$ должна проходить через точки $(0,1)$ и $\left(\alpha^{2}, 0\right)$, т.е. $r\left(L_{2}, L_{4}, L_{10}\right)=2$ и $r\left(L_{3}, L_{8}, L_{10}\right)=2$.

Наконец, потребуем, чтобы прямые $L_{9}$ и $L_{10}$ были параллельны (т.е. $r\left(L_{1}, L_{9}, L_{10}\right)$ $=2)$ : точка пересечения, покрытая "черной дырой” на рис. 7 , должна лежать на бесконечности. Это возможно, только если $\alpha^{4}=1$. Но $\alpha \neq 1$ (поскольку $L_{5} \neq L_{4}$ ) и $\alpha^{2} \neq 1$ (поскольку $L_{8} \neq L_{4}$ ). Следовательно, любая комплексная реализация этого матроида соответствует числу $\alpha$ такому, что $\alpha^{2}=-1$.

\section{$\S$ 15. Приложения в интегральной геометрии: общие гипергеометрические функции}

Теория общих (или многомерных) гипергеометрических функиий была инициирована К. Аомото [1], [2] и И. М. Гельфандом [34]. Эти функции образуют широкий класс функций, обладающих интегральными представлениями: они представляют все такие функции в той же мере, в какой наборы плоскостей представляют класс всех алгебраических множеств. 


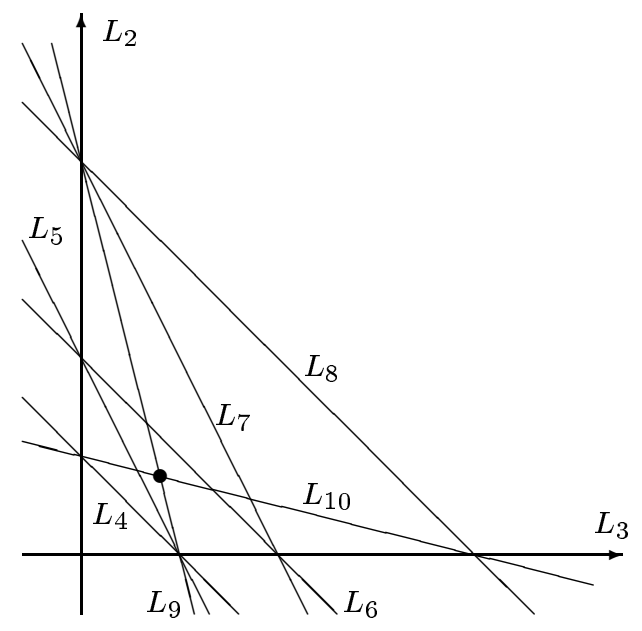

Рис. 7. Мнимый набор прямых

Исходная точка этой теории - это гипергеометрический интеграл Гаусса

$$
\Gamma\left(a ; \alpha_{1}, \alpha_{2}, \alpha_{3}\right) \equiv \int_{0}^{1} z^{\alpha_{1}}(z-1)^{\alpha_{2}}(z-a)^{\alpha_{3}} d z
$$

где $\alpha_{j}$ и $a-$ комплексные числа, $a \notin[0,1]{ }^{1}$

Этот интеграл абсолютно сходится при $\operatorname{Re} \alpha_{1}>-1, \operatorname{Re} \alpha_{2}>-1$. Для любого фиксированного $a$ он является голоморфной функцией в области пространства $\mathbb{C}^{3}$ показателей $\alpha=\left(\alpha_{1}, \alpha_{2}, \alpha_{3}\right)$, выделенной этими неравенствами. Аналитическое продолжение этой функщии на пространство всех $\alpha \in \mathbb{C}^{3}$ является однозначной мероморфной функцией, полюса которой лежат на гиперплоскостях, на которых либо $\alpha_{1}$, либо $\alpha_{2}$ является отрицательньм целым числом.

$\mathrm{C}$ другой стороны, зафиксировав $\alpha$ и меняя $a$, мы получаем аналитическую функцию от $a$ с ветвлением в точках 0 и 1 , удовлетворяюшую знаменитьм гипергеометрическим уравнениям, см. [11]. Если показатели $\alpha$ - обшего положения, то пространство решений этих уравнений в окрестности каждой точки $a$ двумерно и линейно порождено разньми ветвями аналитического продолжения этой функции.

Гораздо более общим образом, можно рассмотреть интеграл

$$
\Gamma(\lambda ; \alpha)=\int_{\Delta} f_{1}^{\alpha_{1}} \cdots f_{k}^{\alpha_{k}} d z_{1} \wedge \cdots \wedge d z_{n}
$$

где $f_{j}$ - некоторые полиномиальные функции $\mathbb{C}^{N} \rightarrow \mathbb{C}$, аналитически зависяшие от параметров $\lambda \in \mathbb{C}^{m}, \alpha_{j}$ - комплексные показатели, а интегрирование ведется по некоторым относительно замкнутым (т.е. локально конечным) но, вообще говоря, не компактньм (т.е. не конечным) $n$-мерным циклам в пространстве ненулевых значений подынтегральной функции (или, точнее, в пространстве некоторого накрытия над этим пространством, в котором наша функция становится однозначной).

Вот один важньй класс таких задач. Зафиксируем некоторьй матроид и разрешим функциям $f_{j}$ быть любьми линейньми функциями $\mathbb{C}^{N} \rightarrow \mathbb{C}$, множества нулей которых образуют всевозможные комплексные реализации этого матроида.

\footnotetext{
${ }^{1}$ Сам Гаусс писал этот интеграл в совсем другом, но эквивалентном виде.
} 
Интеграл (21) также задает мероморфную функцию от $\alpha$ (при фиксированных $a$ ), cp. [12], и ветвящуюся аналитическую функцию от $\lambda$ при фиксированных $\alpha$; множество ее ветвления - это множество таких значений параметра $\lambda$, при которых соответствующие функции $f_{j}$ имеют в совокупности "нетипичные” множества нулей (т.е. функция ранга $r$ набора плоскостей "подскакивает").

Вновь основные задачи здесь - следуюшие:

1) описать множество полюсов этих интегралов, рассматриваемых как мероморффные функции от $\alpha$;

2) описать ветвление этих интегралов при фиксированных $\alpha$ и меняюшихся параметрах $\lambda$ множества функций $f_{j}$;

3) найти число линейно независимых функций на пространстве параметров $\mathbb{C}^{m}$, заданных такими интегралами.

Решение этих аналитических проблем тесно связано со следуюшими топологическими:

А) вычисление групп гомологий, связанных с нашими наборами функций $f_{j}$ и содержаших все возможные контуры интегрирования $\Delta$;

Б) изучение отображений типа (18) для таких групп;

В) изучение топологии (в особенности фундаментальной групшы) конфигурационного пространства всех реализаций нашего матроида;

Г) изучение гомологического векторного расслоения над этим конфигурационным пространством, слоем которого над набором гиперплоскостей является соответствующая группа гомологий, рассмотренная в А); в особенности изучение представления монодромии фундаментальной группы из В) в слоях этого расслоения.

Рассмотрим все эти задачи (и их приложения) для классического интеграла (20). В этом случае решение задачи 1 ) вытекает из рис. 6а): если $\alpha_{1}$ и $\alpha_{2}$ не целые, то наш несобственньй интеграл по интервалу $(0,1)$ можно заменить на аналогичньй интеграл по “двойной петле”, взятый с коэффициентом $1 /\left(1-e^{2 \pi i \alpha_{1}}\right)\left(1-e^{2 \pi i \alpha_{2}}\right)$, а следовательно, неособьй.

Аналогично в общем случае интеграла (21), если набор показателей $\tau_{j} \equiv e^{2 \pi i \alpha_{j}}$ нерезонансньй в смысле $\S 13$, то любой контур интегрирования можно регуляризовать при помоши "многомерной двойной петли", см. [86].

ЗАмЕчАнИЕ. Последнее утверждение не является формальным следствием предложения 8, т.е. обратимости отображения (18) для нерезонансных показателей. Действительно, при изучении интегралов как функций от этих показателей контуры интегрирования нельзя рассматривать как элементы групшы

$$
H_{N}^{\mathrm{lf}}\left(\mathbb{C}^{N} \backslash L, \Theta\right)
$$

поскольку определение этой группы включает факторизацию, зависящую от этих показателей. Тем не менее, геометрическая конструкция “двойных петель” работает и в этом случае и позволяет нам регуляризовать интегралы.

Напротив, если мы фиксируем показатели $\alpha_{j}$, то мы можем рассматривать контуры интегрирования как элементы группы (22). Размерность пространства разных интегральных функций (рассматриваемых как функции только от параметров $\lambda$ функций $f_{j}$ ) не превосходит размерность этой группы. Эта оценка может не достигаться в некоторых экзотических примерах, однако для многих важных матроидов она точна. В частности, это так для матроидов, соответствуюших типичньм наборам и, более 
того, всем наборам с нормальными пересечениями в $\mathbb{C}^{N}$, но, вообше говоря, не в $\mathbb{C P}^{N}$, cм. [88].

Доказательство (см. [86]) основано на изучении действия монодромии в гомологическом расслоении: начиная с единственного контура интегрирования и действуя на него всеми элементами групш монодромии, мы можем получить набор циклов, порождаюших всю групу (22).

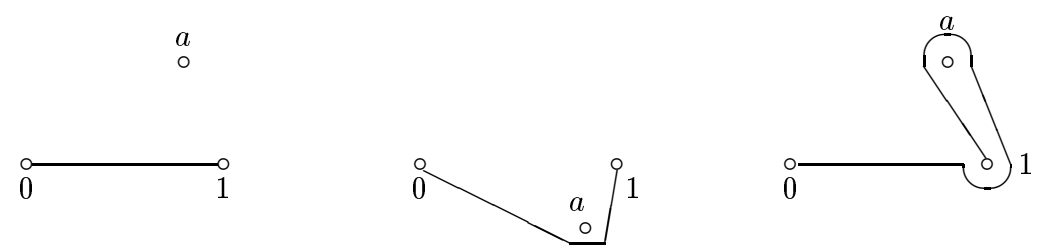

Рис. 8. Монодромия гипергеометрического интеграла

ПРИМЕР. В случае интеграла (20) конфигурационное пространство - это проколотая плоскость $\mathbb{C}^{1} \backslash\{0,1\}$ всех допустимых значений параметра $a$. Проташим это значение вдоль замкнутой петли в этом пространстве, обходящей вокруг некоторой особой точки, скажем, вокруг точки 1. Деформируя одновременно контур интегрирования $(0,1)$ таким образом, чтобы ни в какой момент он не пересекал особых значений 0,1 и текущего значения $a$, мы получим цикл, указанньй на рис. 8 справа. Интеграл типа (20) вдоль этого цикла равен аналитическому продолжению исходного интеграла $(20)$ вдоль нашей петли в пространстве параметров. Разность между этим циклом и исходньм равна интервалу $(1, a)$, пройденному в двух противоположных направлениях на разных листах римановой поверхности, на которой наша форма интегрирования однозначна. Если число $\alpha_{3}$ не целое, то интеграл вдоль этого цикла не равен тождественно нулю, и мы получаем вторую интегральную функцию, независимую от первой. Поскольку размерность групшы $H_{1}^{\mathrm{lf}}\left(\mathbb{C}^{1} \backslash\{0,1, a\}, \Theta\right)$ равна 2 , верхняя и нижняя оценки числа линейно независимых интегральных функций совпали и задача 3 ) решена в этом частном случае.

Об алгебраических свойствах аналитических функций этого типа см., в частности, $[27],[39]$.

Аналогичные (хотя и гораздо более сложные) методы позволяют доказьвать сходные результаты в многомерных ситуациях, см. [88], [86]. В частности, если множества $\left\{x \mid f_{j}(x)=0\right\}$ образуют наборы гиперплоскостей с нормальными пересечениями в $\mathbb{C}^{N}$ и набор показателей $\tau_{j} \equiv e^{2 \pi i \alpha_{j}}$ нерезонансный, то гомологическая оценка точна, т.е. мы имеем максимально возможное число линейно независимых интегральных функций.

Эти и подобные им функции имеют замечательные приложения в математической физике, см. например [67], [73].

\section{$\S$ 16. Что, если набор плоскостей бесконечен?}

Многие важные топологические пространства в $\mathbb{R}^{N}$ представимы в виде непрерьвных семейств плоскостей. Неисчерпаемым источником таких пространств является теория дискриминантов, см. [74], [84]. Описанная выше стратегия топологического исследования объединений плоскостей (и дополнений к таким объединениям), основанная на симплициальных разрешениях, работает и в этих случаях (после подходя- 
щей модификации). В частности, нам приходится рассматривать непрерывные порядковые комплексы и строить конические разрешения таких пространств. Здесь мы опишем простой пример: детерминантное множество.

Пусть $\mathbb{K}$ - поле $\mathbb{R}$ или $\mathbb{C}$ или тело кватернионов $\mathbb{H}$. Детерминантное множество $\operatorname{Det}\left(\mathbb{K}^{n}\right) \subset \operatorname{End}\left(\mathbb{K}^{n}\right) \sim \mathbb{K}^{n^{2}}$ состоит из всех вырожденных операторов $\mathbb{K}^{n} \rightarrow \mathbb{K}^{n}$.

Мы построим разрешение этого множества, даюшее вычисление их групп гомологий Бореля-Мура, а также, при посредстве двойственности Александера, и самое сложное вычисление групп когомологий дополнительных к ним пространств $G L(\mathbb{K}, n)$.

Тавтологическое разрешение множества $\operatorname{Det}\left(\mathbb{K}^{n}\right)$ задается исключением кванторов (которое является аналогом “взятия множеств $S_{j}$ по отдельности" в обосновании формулы включений-исключений). А именно, оператор $A$ принадлежит $\operatorname{Det}\left(\mathbb{K}^{n}\right)$, если существует точка $x \in \mathbb{K}^{n-1}$ такая, что $\{x\} \subset \operatorname{ker} A$. Определим пространство разрешения $\operatorname{det}_{1}\left(\mathbb{K}^{n}\right)$ как пространство всех пар $(x, A) \in \mathbb{K}^{n-1} \times \operatorname{End}\left(\mathbb{K}^{n}\right)$ таких, что $\{x\} \in \operatorname{ker} A$. Это пространство обладает (тавтологической) структурой $\left(n^{2}-n\right)$-мерного $\mathbb{K}$-векторного расслоения над $\mathbb{K} \mathbb{P}^{n-1}$, слой $L(x)$ которого состоит из всех $A$ таких, что $\{x\} \in \operatorname{ker} A$. Очевидная проекция $\pi: \operatorname{det}_{1}\left(\mathbb{K}^{n}\right) \rightarrow \operatorname{Det}\left(\mathbb{K}^{n}\right)$ peгулярна над операторами с одномерными ядрами, а прообраз оператора с $\operatorname{dim} \mathrm{ker}=l$ изоморфен $\mathbb{K}^{l-1}$.

Эта ситуащия аналогична тому, что мы имели вьше: множество $\operatorname{Det}(\mathbb{K}, n)$ является объединением пространств $L(x)$, подобно тому как пространство $L$ было объединением плоскостей $L_{i}$. Сохраняя аналогию, мы построим порядковьй комплекс всех пересечений этих пространств $L(x)$. Это делается не непосредственно, поскольку семейство плоскостей $L(x)$ не дискретно, и, более того, множество таких плоскостей, проходяших через одну и ту же точку множества $\operatorname{Det}\left(\mathbb{K}^{n}\right)$, может быть непрерьвным. Действительно, возможные пересечения нескольких плоскостей $L\left(x_{j}\right) \subset \operatorname{End}\left(\mathbb{K}^{n}\right)-$ это просто плоскости вида $L(X)$, где $X$ - это подпространство некоторой размерности в $\mathbb{K}^{n}$ (т.е. точка некоторого многообразия Грассмана $G_{i}\left(\mathbb{K}^{n}\right), i \in[1, n]$ ), а $L(X)$ состоит из всех операторов, ядра которых содержат $X$.

Поэтому наше частично упорядоченное множество всех плоскостей и их пересечений - это несвязное обгединение всех грассмановых многообразий $G_{1}\left(\mathbb{K}^{n}\right), \ldots$, $G_{n-1}\left(\mathbb{K}^{n}\right), G_{n}\left(\mathbb{K}^{n}\right)$. Непрерывный порядковый комплекс всех этих грассманианов определяется следующим образом. Рассмотрим джойн $G_{1}\left(\mathbb{K}^{n}\right) * \cdots * G_{n}\left(\mathbb{K}^{n}\right)$, т.е., грубо говоря, естественным образом топологизированное объединение симплексов, вершины которых соответствуют точкам разных грассманианов. Такой симплекс назовем когерентным, если плоскости, соответствуюшие его вершинам, образуют флаг. Искомьй порядковьй комплекс $\Xi\left(\mathbb{K}^{n}\right)$ является объединением всех когерентных симплексов, с топологией, индуцированной из топологии джойна. Он является конусом с вершиной $\left\{\mathbb{K}^{n}\right\} \in G_{n}\left(\mathbb{K}^{n}\right)$. Его линк $\partial \Xi\left(\mathbb{K}^{n}\right)$ - это объединение когерентных симплексов, не содержащих вершины $\left\{\mathbb{K}^{n}\right\}$.

Этот линк $\partial \Xi\left(\mathbb{K}^{n}\right)$ гомеоморфен сфиере $S^{M}, M=\frac{1}{2} n(n-1)\left(\operatorname{dim}_{\mathbb{R}} \mathbb{K}\right)+n-2$. (Вероятно, этот факт подразумевается в замечании 1.4 работы [20], см. также [85], [84].) Поэтому $\Xi\left(\mathbb{K}^{n}\right)$ гомеоморфно шару.

Коническое разрешение множества $\operatorname{Det}\left(\mathbb{K}^{n}\right)$ строится как подмножество прямого произведения $\Xi\left(\mathbb{K}^{n}\right) \times \operatorname{Det}\left(\mathbb{K}^{n}\right)$. Любой плоскости $X \subset \mathbb{K}^{n}$ соответствует подпространство $\Xi(X) \subset \Xi\left(\mathbb{K}^{n}\right)$, именно, объединение всех когерентных симплексов, все вершины которых соответствуют плоскостям, лежашим в $X$. Оно является конусом с вершиной $\{X\}$ и гомеоморфно замкнутому шару. Определим коническое раз- 
решение $\delta\left(\mathbb{K}^{n}\right) \subset \Xi\left(\mathbb{K}^{n}\right) \times \operatorname{Det}\left(\mathbb{K}^{n}\right)$ как объединение произведений $\Xi(X) \times L(X)$ по всем плоскостям $X$ размерностей $1, \ldots, n$. Легко видеть, что очевидная проекция $\delta\left(\mathbb{K}^{n}\right) \rightarrow \operatorname{Det}\left(\mathbb{K}^{n}\right)$ индуцирует гомотопическую эквивалентность одноточечных компактификаций этих пространств (действительно, это проектирование собственно и полуалгебраично, и все его слои являются стягиваемыми конусами вида $\Xi(X))$. $\mathrm{C}$ другой стороны, пространство $\delta\left(\mathbb{K}^{n}\right)$ обладает удобной фильтрацией: член $F_{i}$ ее - это объединение произведений $\Xi(X) \times L(X)$ по всем плоскостям $X$ размерностей $\leqslant i$. Член $F_{i} \backslash F_{i-1}$ этой фильтрации является тотальным пространством расслоения над $G_{i}\left(\mathbb{K}^{n}\right)$. Его слой над точкой $\{X\}$ - это пространство $(\Xi(X) \backslash \partial \Xi(X)) \times L(X)$, оно гомеоморфно евклидову пространству. Следовательно, группа гомологий Бореля-Мура этого члена сводится к аналогичной группе базы. Спектральная последовательность, порожденная этой фильтрацией и сходящаяся к групе гомологий Бореля-Мура множества $\operatorname{Det}\left(\mathbb{K}^{n}\right)$ (или, эквивалентно, к группе когомологий дополнительного пространства $\left.G L\left(\mathbb{K}^{n}\right)\right)$, стабилизируется в первом члене (т.е. $E_{1}^{p, q} \equiv E_{\infty}^{p, q}$ ) и дает, в частности, доказательство гомологического расщепления Muллера

$$
H_{m}\left(G L\left(\mathbb{C}^{n}\right)\right)=\bigoplus_{k=0}^{n} H_{m-k^{2}}\left(G_{k}\left(\mathbb{C}^{n}\right)\right)
$$

и аналогичных расшеплений для $\mathbb{K}=\mathbb{R}$ и $\mathbb{H}$.

Имеется множество других проблем, в которых работает технология конических разрешений, см. [80], [82]. Среди них - теория узлов и типичных плоских кривых (см. следующий параграф), топологическое исследование пространств непрерьвных отображений, гладких функций без сложных особенностей, операторов с простыми спектрами, неособых проективных гиперповерхностей ...

\section{§17. Приложения и аналогии в дифференциальной топологии}

Пространство $M(N, 2)$ (см. $\S 2)$ можно рассматривать как пространство всех вложений в $\mathbb{C}^{1}$ конечного множества мошности $N$. Аналогичным образом мы можем рассмотреть пространство всех гладких вложений $S^{1} \hookrightarrow \mathbb{R}^{n}$, т.е. ручных узлов в $\mathbb{R}^{n}$; если $n=3$, то 0 -мерные классы когомологий этого пространства - это инварианты узлов. Мы можем изучать эти и прочие классы когомологий (в случае произвольного $n \geqslant 3$ ) по сушеству теми же методами, что в $\S \S 7,8$ (забавно, что в действительности это было сделано раньше, см. [75]). Мы рассматриваем пространство $\mathscr{K}$ всех гладких отображений $S^{1} \rightarrow \mathbb{R}^{n}$, определяем дискриминант $\Sigma \subset \mathscr{K}$ как пространство всех отображений, не являюшихся гладкими вложениями, и изучаем группу $H^{*}(\mathscr{K} \backslash \Sigma)$. Для Этого мы строим коническое разрешение дискриминантного множества $\Sigma$. Это возможно, поскольку это множество заметается разумным семейством подпространств в $\mathscr{K}$. Эти подпространства параметризуются всеми неупорядоченными парами точек

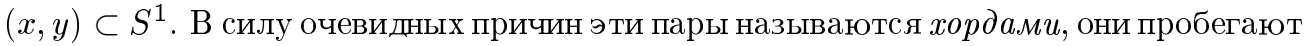
двумерное многообразие $\overline{B\left(S^{1}, 2\right)}$.

Для любой такой пары соответствуюшее пространство $L(x, y)$ состоит из всех отображений $f: S^{1} \rightarrow \mathbb{R}^{n}$ таких, что $f(x)=f(y)$, если $x \neq y$, или $f^{\prime}(x)=0$, если $x=y$. Такие пространства образуют тавтологическое разрешение множества $\Sigma$. Затем мы берем порядковьй комплекс всех возможных пересечений

$$
L\left(x_{1}, y_{1}\right) \cap L\left(x_{2}, y_{2}\right) \cap \cdots
$$


ТАБлицА 1. Аналогия между теорией наборов плоскостей и теорией узлов

\begin{tabular}{|c|c|}
\hline Теория наборов плоскостей & Теория узлов \\
\hline Пространство $\mathbb{R}^{N}$ & Пространство $\mathscr{K}=C^{\infty}\left(S^{1}, \mathbb{R}^{n}\right)$ \\
\hline Носитель набора $L=\bigcup L_{i} \subset \mathbb{R}^{N}$ & Дискриминант $\Sigma \subset \mathscr{K}$ \\
\hline Множество индексов $\{1, \ldots, m\}$ & Пространство хорд $\overline{B\left(S^{1}, 2\right)}$ \\
\hline Плоскость $L_{i}$ & Подпространство $L(x, y), x, y \in S^{1}$ \\
\hline Несвязное объединение $\bigsqcup L_{i}$ & Тавтологическое разрешение множества $\Sigma$ \\
\hline Симплициальное разрешение $L^{\prime}$ & Коническое разрешение $\sigma$ дискриминанта $\Sigma$ \\
\hline $\begin{array}{l}\text { Подмножества } I \subset\{1, \ldots, m\} \\
c \operatorname{codim} L_{I}=p\end{array}$ & $\begin{array}{l}\text { Комбинаторные типы хордовых } \\
\text { конфигураций } J \text { c codim } L(J)=p n\end{array}$ \\
\hline Призма $L_{I}^{\prime}$ & $J$-блок в $\sigma$ \\
\hline $\begin{array}{l}\text { Формула тасовочного } \\
\text { произведения из } \S 11\end{array}$ & $\begin{array}{l}\text { Формулы умножения Концевича (для инвариантов) } \\
\text { и Турчина (для старших когомологий) }\end{array}$ \\
\hline $\begin{array}{l}\text { Дерамовские } \\
\text { представления из } \S \S 3,4\end{array}$ & Интеграл Концевича \\
\hline Расщепление (12) & Теорема Концевича о стабилизации \\
\hline $\begin{array}{l}\text { Комбинаторная } \\
\text { реализация из } § 10\end{array}$ & $\begin{array}{l}\text { Комбинаторные формулы для инвариантов } \\
{[63],[42] \text { и прочих классов когомологий [81] }}\end{array}$ \\
\hline
\end{tabular}

и предельных положений таких пересечений (все они суть подпространства в $\mathscr{K}$, коразмерности которых кратны $n$ ), снабжаем его подходящей топологией и определяем коническое разрешение точно так же, как ранњше, т.е. как подмножество прямого произведения этого порядкового комплекса на пространство $\mathscr{K}$. Затем мы определяем фильтрацию на этом разрешении в соответствии с коразмерностями (деленными на $n$ ) этих плоскостей и рассматриваем возникающую спектральную последовательность.

Гомологическое изучение получаюшегося разрешения известно как теория инвариантов конечного порядка для узлов, см. [10], и различные ее обобщения, включая (столь же интересное) вычисление старших когомологий пространств узлов, см. [75], [83], [69]. В таблище 1 дан краткий список параллельных понятий и объектов в обеих теориях. Конечно, значительная часть ее правой половины не может быть здесь объяснена: эта таблица скорее представляет собой разновидность Розеттского камня для комбинаторщиков, которые захотели бы изучить теорию инвариантов узлов, см. [10].

Необходимо отметить также исключительное значение тождества Арнольда (3) для конструкции интеграла Конщевича [48], [49].

Конечно, пространство $\mathscr{K}$ бесконечномерно, и формально мы не можем применять двойственность Александера в нем: обычные (т.е. конечномерные) классы когомологий пространства узлов $\mathscr{K} \backslash \Sigma$ должны соответствовать "бесконечномерньм циклам" в $\Sigma$, определение которых требует дополнительных усилий. Строгое построение таких циклов, соответствующих классам когомологий конечного типа, использует технику конечномерных аппроксимаций, см. [75]. 
Аналогично, мы можем рассмотреть пространство гладких вложений конечного набора окружностей в $\mathbb{R}^{n}$, это дает теорию классов когомологий конечного типа в пространстве зацеплений.

Еще одно пространство этого типа - пространство всех плоских кривых без тройных точек, см. [3], [6], [76], [77], [52]-[55], [68], [46], [79]. Оно тесно связано с наборами плоскостей $A(N, 3)$, см. [17] и $\S 2$.

Я благодарю всех, кому я обязан познаниями в описанной области, в том числе В. И. Арнольда, А. Бьёрнера, Ф. Велкера, А. М. Вершика, И. М. Гельфанда, Р. Т. Живалевича, А. В. Зелевинского, М. М. Капранова, А. Б. Меркова, Н. Е. Мнёва, С. А. Юзвинского, Г. Л. Рыбникова, В. В. Серганову, В. Э. Турчина, Г. Циглера, Б. З. Шапиро и К. Шульц.

\section{СПИСОК ЛИТЕРАТУРЫ}

[1] K. Aomoto. Les équations aux différences linéaires et les intégrales des fonctions multiformes // J. Fac. Sci. Univ. Tokyo Sect. IA Math. 1975. V. 22. P. 271-297.

[2] K. Aomoto. On the structure of integrals of power products of linear functions // Sci. Papers College Gen. Ed. Univ. Tokyo. 1977. V. 27. P. 49-61.

[3] V.I. Arnold. Plane curves, their invariants, perestroikas and classifications // Singularities and Bifurcations / ed. V. I. Arnold. Providence, RI: Amer. Math. Soc., 1994. P. 33-91.

[4] В.И. Арнольд. Кольцо когомологий группы крашеных кос // Матем. заметки. 1969. T. 5. № 2. C. $227-231$.

[5] В.И. Арнольд. О некоторых топологических инвариантах алгебраических функций // Труды ММО. 1970. Т. 21. С. 27-46.

[6] В.И. Арнольд. Инварианты и перестройки фронтов на плоскости // Труды МИАН. 1995. T. 209. C. $14-64$

[7] В.И. Арнольд, В. А. Васильев, В. В. Горюнов, О. В. Ляшко. Особенности. II. Приложения // Итоги науки и техн. Фундам. напр. Т. 6. М.: ВИНИТИ, 1989.

[8] E. Artin. Theorie der Zöpfe // Abh. Math. Sem. Univ. Hamburg. 1925. V. 4. P. 47-72. (English transl.: Theory of braids. Ann. of Math. (2). 1947. V. 48. № 1. P. 101-126.)

[9] E. Babson, A. Björner, S. Linusson, J. Shareshian, V. Welker. The complexes of not $i$-connected graphs // Topology. 1999. V. 38. № 2. P. 271-299.

[10] D. Bar-Natan. Bibliography of Vassiliev Invariants // http://www.ma.huji.ac.il/ drorbn /VasBib/VasBib.html.

[11] Г. Бейтмен, А. Эрдейи. Высшие трансцендентные функции. М.: Наука, 1965.

[12] И. Н. Бернштейн, С. И. Гельфанд. Мероморфность функции $P^{\lambda} / /$ Функц. анализ и его прил. 1969. Т. 3. № 4. С. 84-85.

[13] A. Björner, M. Las Vergnas, B. Sturmfels, N. White, G. M. Ziegler. Oriented Matroids. London: Cambridge Univ. Press, 1992.

[14] A. Björner. Subspace arrangements // Proceedings of the 1-st European Congress of Mathematicians (Paris, 1992). V. 1 / ed. A. Joseph et al. Basel: Birkhäuser, 1994. P. 321-370. (Progr. Math. V. 119.)

[15] A. Björner. Topological methods // Handbook of Combinatorics / ed. R. Graham, M. Grötschel, L. Lovasz. Amsterdam: North-Holland, 1995. P. 1819-1872.

[16] A. Björner. Nonpure shellability, $f$-vectors, subspace arrangements and complexity // Formal Power Series and Algebraic Combinatorics (New Brunswick, NJ, 1994) / ed. L. Billera et al. Providence, RI: Amer. Math. Soc., 1996. P. 25-53. (DIMACS Ser. Discrete Math. Theoret. Comput. Sci. V. 24.)

[17] A. Björner, V. Welker. The homology of " $k$-equal" manifolds and related partition lattices // Adv. Math. 1995. V. 110. № 2. P. 277-313.

[18] A. Björner, G. M. Ziegler. Combinatorial stratification of complex arrangements // J. Amer. Math. Soc. 1992. V. 5. № 1. P. 105-149.

[19] A. Björner, L. Lovász, A. Yao. Linear decision trees: volume estimates and topological bounds // Proceedings of the 24-th Annual ACM Symposium on Theory of Computing. New York: ACM Press, 1992. P. 170-177. 
[20] A. Borel, J.-P. Serre. Cohomologie d'immeubles et de groupes $S$-arithmétiques // Topology. 1976. V. 15. P. 211-231.

[21] Н. Бурбаки. Группы и алгебры Ли. М.: Мир, 1972.

[22] Э. Брискорн. О группах кос (по В.И. Арнольду) // Математика. 1974. Т. 18. № 3. C. $46-59$.

[23] P. Cartier. Les arrangements d'hyperplans: un chapitre de géométrie combinatoire // Séminaire Bourbaki 1980/81. Berlin: Springer-Verlag, 1981. P. 1-22. (Lecture Notes in Math. V. 901.)

[24] C. De Concini, C. Procesi. Wonderful models of subspace arrangements // Selecta Math. (N.S.). 1995. V. 1. № 3. P. 459-494.

[25] P. Deligne. Equations différentielles à points singuliers réguliers. Berlin: Springer-Verlag, 1970. (Lect. Notes in Math. V. 163.)

[26] P. Deligne. Les immeubles des groupes de tresses généralisés // Invent. Math. 1972. V. 17. P. 273-302.

[27] P. Deligne, G. Mostow. Monodromy of hypergeometric functions and nonlattice integral monodromy // Inst. Hautes Études Sci. Publ. Math. 1986. V. 63. P. 5-89.

[28] P. Deligne, M. Goresky, R. MacPherson. L'algèbre de cohomologie du complément, dans un espace affine, d'une famille finie de sous-espaces affines // Michigan J. Math. 2000. V. 48.

[29] M. de Longueville, C. Schultz. The cohomology rings of complements of subspace arrangements // http://www.math.fu-berlin.de/ ${ }^{\sim}$ cschultz/arrangements.html; (to appear).

[30] H. Esnault, V. Shechtman, E. Viehweg. Cohomology of local systems on the complement of hyperplanes // Invent. Math. 1992. V. 109. № 3. P. 557-561; 1993. V. 112. № 2 . P. 447.

[31] E. Fadell, L. Neuwirth. Configuration spaces // Math. Scand. 1962. V. 10. № 1. P. 111-118.

[32] J. Folkman. The homology groups of a lattice // J. Math. Mech. 1966. V. 15. P. 631-636.

[33] Д. Б. Фукс. Когомологии группы кос $\bmod 2 / /$ Функц. анализ и его прил. 1970. Т. 4. № 2. C. $62-73$.

[34] И. М. Гельфанд. Общая теория гипергеометрических функций // Докл. АН СССР. 1986. T. 288. №1. С. $14-18$.

[35] И. М. Гельфанд, С. И. Гельфанд. Обобщенные гипергеометрические уравнения // Докл. АН СССР. 1986. Т. 288. № 2. С. 279-283.

[36] И. М. Гельфанд, Г. Л. Рыбников. Алгебраические и топологические инварианты ориентированных матроидов // Докл. АН СССР. 1989. Т. 307. № 4. С. 791-795.

[37] И. М. Гельфанд, В.В.Серганова. Комбинаторные геометрии и страты тора на однородных компактных многообразиях // УМН. 1987. Т. 42. № 2. С. 107-134.

[38] И. М. Гельфанд, А. В. Зелевинский. Алгебраические и комбинаторные аспекты общей теории гипергеометрических функций // Функц. анализ и его прил. 1986. Т. 20. № 3. C. $17-34$.

[39] I. M. Gelfand, M. M. Kapranov, A. V. Zelevinskii. Discriminants, Resultants, and Multidimensional Determinants. Boston, MA: Birkhäuser, 1994; И. М. Гельфанд, M.M. Капранов, А. В. Зелевинский. Дискриминанты, резултанты и многомерные детерминанты. М.: Фазис (в печати).

[40] М. Горески, Р. Макферсон. Стратифицированная теория Морса. М.: Мир, 1991.

[41] В. В. Горюнов. Когомологии групп кос серий $C$ и $D / /$ Труды ММО. 1981. Т. 42. C. $234-242$.

[42] M. Goussarov, M. Polyak, O. Viro. Finite type invariants of classical and virtual knots // Topology. 2000. V. 39. № 5. P. 1045-1068.

[43] A. Hattori. Topology of $\mathbf{C}^{n}$ minus a finite number of affine hyperplanes in general position // J. Fac. Sci. Univ. Tokyo Sect. IA Math. 1975. V. 22. P. 205-219.

[44] Х. Хиронака. Разрешение особенностей алгебраических многообразий над полями характеристики нуль // Математика. 1965. Т. 9. №6. С. 2-70; 1966. Т. 10. №1. С. 3-89; T. 10. № 2. C. 3-58.

[45] K. Jewell, P. Orlik, B. Z. Shapiro. On the complement of affine subspace arrangements // Topology Appl. 1994. V. 56. P. 215-233.

[46] M. Khovanov. Doodle groups // Trans. Amer. Math. Soc. 1996. V. 349. P. 2297-2315. 
[47] M. Khovanov. Real $K(\pi, 1)$ arrangements from finite root systems // Math. Res. Lett. 1996. V. 3. P. 261-274.

[48] M. Kontsevich. Vassiliev's knot invariants // Adv. Soviet Math. 1993. V. 16, part 2. P. $137-150$.

[49] M. Kontsevich. Formal (non-)commutative symplectic geometry // The I. M. Gel'fand Mathematical Seminars 1990-1992 / ed. L. Corwin et al. Basel: Birkhäuser, 1993. P. 173-187.

[50] В.Я. Лин. Косы Артина и связанные с ними группы и пространства // Итоги науки и техн. Алгебра, Топология, Геометрия. Т. 17. М.: ВИНИТИ, 1979. С. 159-227.

[51] S. MacLane. Some interpretations of abstract linear dependence in terms of projective geometry // Amer. J. Math. 1936. V. 58. P. 236-240.

[52] A. B. Merkov. Finite order invariants of ornaments // J. Math. Sci. 1998. V. 90. № 4. P. 2215-2273.

[53] A. B. Merkov. Vassiliev invariants classify flat braids // Differential and Symplectic Topology of Knots and Curves / ed. S.L. Tabachnikov. Providence, RI: Amer. Math. Soc., 1999. P. 83-102. (Amer. Math. Soc. Transl. Ser. 2. V. 190.)

[54] A. B. Merkov. Vassiliev invariants classify plane curves and doodles // Preprint, 1998,

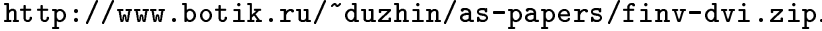

[55] А. Б. Мерков. Сегментно-стрелочные диаграммы и инварианты орнаментов // Матем. сб. 2000. Т. 191. № 11. С. 47-78.

[56] Н. Е. Мнёв. О многообразиях комбинаторных типов проективных конфигураций и выпуклых многогранников // Докл. АН СССР. 1985. Т. 283. № 6. С. 1312-1314.

[57] N.E. Mnev. The universality theorems on the classification problem of configuration varieties and convex polytopes varieties // Topology and Geometry - Rohlin Seminar / ed. O. Viro. Berlin: Springer-Verlag, 1988. P. 527-544. (Lecture Notes in Math. V. 1346.)

[58] T. Nakamura. The topology of the configuration of projective subspaces in a projective space. I; II // Sci. Papers College Arts Sci. Univ. Tokyo. 1987. V. 37. P. 13-35; 1991. V. 41. № 2. P. 59-81.

[59] P. Orlik, L. Solomon. Combinatorics and topology of complements of hyperplanes // Invent. Math. 1980. V. 56. № 2. P. 167-189.

[60] P. Orlik, H. Terao. Arrangements of Hyperplanes. Berlin: Springer-Verlag, 1992.

[61] Ф. Фам. Обобщенные формулш Пикара-Лефшеца и ветвление интегралов // Математика. 1969. T. 13. № 4. C. $61-93$.

[62] Ф. Фам. Введение в топологическое исследование особенностей Ландау. М.: Мир, 1970.

[63] M. Polyak, O. Viro. Gauss diagram formulas for Vassiliev invariants // Internat. Math. Res. Notices. 1994. V. 11. P. 445-453.

[64] G. L. Rybnikov. On the fundamental group of the complex hyperplane arrangement // math. AG/9805056. 1998.

[65] M. Salvetti. Topology of the complement of real hyperplanes in $\mathbb{C}^{N} / /$ Invent. Math. 1987. V. 88. P. $603-618$

[66] M. Salvetti. The homotopy type of Artin groups // Math. Res. Lett. 1994. V. 1. № 5. P. $565-577$.

[67] V. Schechtman, A. Varchenko. Arrangements of hyperplanes and Lie algebra homology // Invent. Math. 1991. V. 106. P. 139-194.

[68] А. Шумакович. Явные формулы для странности плоской кривой // Алгебра и анализ. 1995. T. 7. № 3. C. 165-199.

[69] V. Tourtchine. Sur l'homologie des espaces des nœuds non-compacts // Preprint, 2000; arXiv:math.QA/0010017.

[70] В.Э. Турчин. Группы гомологий комплексов двусвязных графов // УМН. 1997. Т. 52. № 2. C. $189-190$.

[71] V.E. Turchin. Homology isomorphism of the complex of 2-connected graphs and the graph complex of trees // Topics in Quantum Groups and Finite-Type Invariants. Mathematics at the Independent University of Moscow / ed. B. Feigin, V. Vassiliev. Providence, RI: Amer. Math. Soc., 1998. P. 145-153. (Amer. Math. Soc. Transl. Ser. 2. V. 185.)

[72] А. Н. Варченко. Комбинаторика и расположение аффинных гиперплоскостей в вещественном пространстве // Функц. анализ и его прил. 1987. Т. 21. № 1. С. 11-22.

[73] А. Н. Варченко. Многомерные гипергеометрические функции в конформной теории поля, алгебраической $K$-теории, алгебраической геометрии // Международньй конгресс математиков в Киото, 1990. М.: Мир, 1996. С. 401-428. 
[74] V. A. Vassiliev. Complements of Discriminants of Smooth Maps: Topology and Applications. Providence, RI: Amer. Math. Soc., 1994. (Transl. Math. Monographs. V. 98.)

[75] V. A. Vassiliev. Cohomology of knot spaces // Theory of Singularities and its Applications / ed. V.I. Arnold. Providence, RI: Amer. Math. Soc., 1990. P. 23-69. (Adv. Soviet Math. V. 1.)

[76] V.A. Vassiliev. Complexes of connected graphs // The I.M. Gel'fand Mathematical Seminars 1990-1992 / ed. L. Corvin et al. Basel: Birkhäuser, 1993. P. 223-235.

[77] V. A. Vassiliev. Invariants of ornaments // Singularities and Bifurcations / ed. V.I. Arnold. Providence, RI: Amer. Math. Soc., 1994. P. 225-262. (Adv. Soviet Math. V. 21.)

[78] V. A. Vassiliev. Topology of two-connected graphs and homology of spaces of knots // Differential and Symplectic Topology of Knots and Curves / ed. S. L. Tabachnikov. Providence, RI: Amer. Math. Soc., 1999. P. 253-286. (Amer. Math. Soc. Transl. Ser. 2. V. 190.)

[79] V. A. Vassiliev. On finite-order invariants of triple points free plane curves // D. B. Fuchs Anniversary Volume / ed. A. Astashkevich, S. Tabachnikov. Providence, RI: Amer. Math. Soc., 1999. P. 275-300. (Amer. Math. Soc. Transl. Ser. 2. V. 194.)

[80] V. A. Vassiliev. Topological order complexes and resolutions of discriminant sets // Publ. Inst. Math. (Beograd) (N.S.). 1999. V. 66 (80). P. 165-185.

[81] V.A. Vassiliev. On combinatorial formulas for cohomology of spaces of knots // Moscow Math. J. 2001. V. 1. № 1. P. 91-123.

[82] V.A. Vassiliev. Resolutions of discriminants and topology of their complements // Proceedings of the Advanced Summer Institute on Singularity Theory / ed. D. Siersma et al. Dordrecht: Kluwer, 2001.

[83] V. A. Vassiliev. Homology of spaces of knots in any dimensions // London Roy. Soc. Philos. Trans. A. Proceedings of the Discussion Meeting of the London Royal Society "Topological Methods in the Physical Sciences", 2001.

[84] В. А. Васильев. Топология дополнений к дискриминантам. М.: Фазис, 1997.

[85] В. А. Васильев. Геометрическая реализация гомологий классических групп Ли и комплексы, $S$-двойственные флаговым многообразиям // Алгебра и анализ. 1991. Т. 3. № 4. C. $113-120$.

[86] В. А. Васильев. Ветвящиеся интегралы. М.: МЦНМО, 2000.

[87] В. А. Васильев, В. В. Серганова. О числе вещественных и комплексных модулей особенностей гладких функций и реализаций матроидов // Матем. заметки. 1991. Т. 49. № 1. C. $19-27$.

[88] В. А. Васильев, И. М. Гельфанд, А. В. Зелевинский. Общие гипергеометрические функции на комплексных грассманианах // Функц. анализ и его прил. 1987. Т. 21. № 1. C. $23-38$.

[89] Дж. Уайтхед. Новейшие достижения в теории гомотопий. М.: Мир, 1974.

[90] С. Юзвинский. Алгебры Орлика-Соломона в алгебре и топологии // УМН. 2001. Т. 56. № 2. C. $87-166$.

[91] S. Yuzvinsky. Small rational model of subspace complement // http://xxx.lanl.gov/ abs/math.c0/9806143; (to appear).

[92] S. Yuzvinsky. Rational model of subspace complement on atomic complex // Publ. Inst. Math. (Beograd) (N.S.). 1999. V. 66 (80). P. 157-164.

[93] T. Zaslavsky. Facing up to Arrangements: Face-Count Formulas for Partitions of Space by Hyperplanes. Providence, RI: Amer. Math. Soc., 1975. (Mem. Amer. Math. Soc. V. 154.)

[94] G. M. Ziegler. On the difference between real and complex arrangements // Math. Z. 1993. V. 212. P. 1-11.

[95] G. M. Ziegler. Combinatorial models for subspace arrangements. Habilitations-Schrift. Berlin: Techn. Univ. Berlin, 1992.

[96] G. M. Ziegler, R. T. Živaljević. Homotopy type of arrangements via diagrams of spaces // Math. Ann. 1993. V. 295. P. 527-548. 